\title{
MAGNESIUM ISOTOPIC ABUNDANCE RATIOS IN COOL STARS
}

\author{
DAVID Yong AND DAVID L. LAMBERT \\ Department of Astronomy, University of Texas at Austin, Austin, TX 78712; \\ tofu@astro.as.utexas.edu,dll@astro.as.utexas.edu \\ AND \\ INESE I. IVANS ${ }^{1}$ \\ Astronomy Department, California Institute of Technology, Pasadena, CA 91125; \\ iii@astro.caltech.edu \\ Received 2003 July 23; accepted 2003 August 29
}

\begin{abstract}
From high-resolution spectra of 61 cool dwarfs and giants, $\mathrm{Mg}$ isotopic abundance ratios ${ }^{24} \mathrm{Mg}:{ }^{25} \mathrm{Mg}:{ }^{26} \mathrm{Mg}$ are derived from spectral synthesis of the $\mathrm{MgH} \mathrm{A}-\mathrm{X}$ lines near $5140 \AA$. Our sample spans the range $-2.5 \leq[\mathrm{Fe} / \mathrm{H}] \leq 0.1$, including the first measurements of $\mathrm{Mg}$ isotope ratios in stars with metallicities below $[\mathrm{Fe} / \mathrm{H}]=-2.0$. We confirm the decrease in ${ }^{25} \mathrm{Mg} /{ }^{24} \mathrm{Mg}$ and ${ }^{26} \mathrm{Mg} /{ }^{24} \mathrm{Mg}$ with decreasing $[\mathrm{Fe} / \mathrm{H}]$, as predicted by recent models of Galactic chemical evolution in which the $\mathrm{Mg}$ isotopes are produced in massive stars. A subset of kinematically identified thin-disk stars have $\mathrm{Mg}$ isotope ratios in excellent agreement with the predictions. Within the measurement uncertainties, these thin-disk stars show no scatter about the predictions. Several of our stars are likely members of the thick disk, and their high $\mathrm{Mg}$ isotopic ratios may reflect the nucleosynthetic history of the thick disk, which is distinct from the predictions for, and observations of, the thin disk. For thick-disk and halo stars we find a scatter in ${ }^{25} \mathrm{Mg} /{ }^{24} \mathrm{Mg}$ and ${ }^{26} \mathrm{Mg} /{ }^{24} \mathrm{Mg}$ exceeding our measurement uncertainties and increasing with increasing metallicity. Our data suggest that an additional source of ${ }^{25} \mathrm{Mg}$ and ${ }^{26} \mathrm{Mg}$ is required. Intermediate-mass asymptotic giant branch stars are likely candidates.

Subject headings: Galaxy: abundances — Galaxy: evolution — stars: abundances — stars: late-type — subdwarfs
\end{abstract}

\section{INTRODUCTION}

Studies of the chemical evolution of the Galaxy seek a full understanding of the chemical composition of objects - past and present-throughout the Galaxy. Much of the observational data have been provided by stars now in the solar neighborhood whose ages and places of birth may differ considerably from our Sun's present location and age. Elemental abundances observed in large samples of disk stars (e.g., Edvardsson et al. 1993; Reddy et al. 2003), extremely metal-poor stars (e.g., McWilliam et al. 1995; Ryan, Norris, \& Beers 1996), and the general halo population (e.g., Fulbright 2000) have provided insights into the cycle of star formation, evolution, and death that has controlled the composition of gas in the Galaxy. Theoretical efforts (including Timmes, Woosley, \& Weaver 1995, hereafter TWW1995; Goswami \& Prantzos 2000, hereafter GP2000; and Alibés, Labay, \& Canal 2001, hereafter ALC2001) present predictions of the evolution of elements from carbon to zinc. The basic ingredients in these models of Galactic chemical evolution include stellar yields, the initial mass function (IMF), and the star formation rate. The success of a model can be gauged by how accurately the predictions match the observational data. In turn, the observations can be used to constrain the models. The magnesium isotopes present a rare opportunity whereby the evolution of isotopic abundances can be measured to test directly models of Galactic chemical evolution.

The stable isotopes of $\mathrm{Mg}$ consist of the dominant ${ }^{24} \mathrm{Mg}$ and the neutron-rich minor isotopes ${ }^{25} \mathrm{Mg}$ and ${ }^{26} \mathrm{Mg}$. Massive

\footnotetext{
${ }^{1}$ Hubble Fellow.
}

stars produce ${ }^{24} \mathrm{Mg}$ in their carbon- and neon-burning shells before their deaths as Type II supernovae (Arnett \& Thielemann 1985; Thielemann \& Arnett 1985). Helium burning is responsible for the synthesis of the less abundant isotopes via ${ }^{22} \mathrm{Ne}(\alpha, n)^{25} \mathrm{Mg}(n, \gamma)^{26} \mathrm{Mg}$. The production of the heavier $\mathrm{Mg}$ isotopes relies on the abundance of ${ }^{22} \mathrm{Ne}$, which is set primarily by the initial abundances of $\mathrm{C}, \mathrm{N}$, and $\mathrm{O}$. A consequence of the $\mathrm{H}$-burning $\mathrm{CNO}$ cycle is that in equilibrium, ${ }^{14} \mathrm{~N}$ is the dominant nucleus, whose abundance is essentially equal to the initial abundances of $\mathrm{C}+\mathrm{N}+\mathrm{O}$. Successive alpha captures on ${ }^{14} \mathrm{~N}$ can then produce ${ }^{22} \mathrm{Ne}$, the seed from which ${ }^{25} \mathrm{Mg}$ and ${ }^{26} \mathrm{Mg}$ are synthesized. Therefore, the yields of ${ }^{25} \mathrm{Mg}$ and ${ }^{26} \mathrm{Mg}$ are predicted to increase with increasing metallicity. Yields calculated from massive stars (Woosley \& Weaver 1995) indeed verify the increase of the neutron-rich $\mathrm{Mg}$ isotopes with increasing metallicity.

Beginning with Boesgaard (1968), the relative abundances of the $\mathrm{Mg}$ isotopes have been measured in stars from analyses of MgH lines. Tomkin \& Lambert (1980) provided the first evidence that metal-poor stars possess low ratios of ${ }^{25} \mathrm{Mg} /{ }^{24} \mathrm{Mg}$ and ${ }^{26} \mathrm{Mg} /{ }^{24} \mathrm{Mg}$ from an analysis of the subdwarf $\mathrm{Gmb}$ 1830. Later studies of the stellar Mg isotopes include those conducted by Barbuy (1985), Lambert \& McWilliam (1986), Barbuy, Spite, \& Spite (1987), and McWilliam \& Lambert (1988). Recently, Gay \& Lambert (2000) measured a ratio of ${ }^{24} \mathrm{Mg}:{ }^{25} \mathrm{Mg}:{ }^{26} \mathrm{Mg}$ to $[\mathrm{Fe} / \mathrm{H}]$ as low as $\simeq-1.5$. Gay \& Lambert found reasonable agreement between the measured isotopic ratios and predictions from the TWW1995 model of the solar neighborhood, in which the $\mathrm{Mg}$ isotopes were produced by massive stars. Gay \& Lambert also demonstrated that some stars show convincing and unusual excesses of the heavier $\mathrm{Mg}$ isotopes relative 
to other stars of the same $[\mathrm{Fe} / \mathrm{H}]$. In several of these unusual cases, an s-process enrichment was also evident. This suggests that these peculiar compositions were a direct consequence of contamination of the star (or the star's natal cloud) by ejecta from intermediate-mass asymptotic giant branch (AGB) stars. Sufficiently massive AGB stars experience thermal pulses (He shell flashes), leading to the reaction ${ }^{22} \mathrm{Ne}(\alpha, n)^{25} \mathrm{Mg}$ (e.g., Iben 1975; Forestini \& Charbonnel 1997). The neutrons released from this reaction can then enrich the envelope in $s$-process elements, along with ${ }^{25} \mathrm{Mg}$ and ${ }^{26} \mathrm{Mg}$.

Gay \& Lambert (2000) measured Mg isotopic ratios in 20 stars. Prior to Gay \& Lambert, $\mathrm{Mg}$ isotopic ratios had been measured in about 20 stars. In this study, we report $\mathrm{Mg}$ isotopic abundance ratios for 61 stars. Our measurements extend in metallicity down to $[\mathrm{Fe} / \mathrm{H}]=-2.5$, in order to investigate the evolution of the $\mathrm{Mg}$ isotopic ratios at low metallicities.

\section{OBSERVATIONS AND DATA REDUCTION}

A dedicated search for cool subdwarfs was conducted to provide suitable targets to extend the $\mathrm{Mg}$ isotopic measurements below $[\mathrm{Fe} / \mathrm{H}] \simeq-1.5$; the stars (dwarfs and giants) known to have $[\mathrm{Fe} / \mathrm{H}]<-1.5$ are all too warm to provide $\mathrm{MgH}$ lines of adequate strength to measure the isotopic ratios. For the selection criteria, analysis, and further details regarding the search for cool subdwarfs, see Yong \& Lambert (2003a, hereafter Paper I). For $[\mathrm{Fe} / \mathrm{H}]<-1.5$, the neutron-rich isotopes are only expected to provide a small contribution to the total $\mathrm{Mg}$ abundance, ${ }^{25} \mathrm{Mg} /{ }^{24} \mathrm{Mg}$ and ${ }^{26} \mathrm{Mg} /{ }^{24} \mathrm{Mg}$ both less than 0.05 . The target stars should therefore have strong $\mathrm{MgH}$ lines, in order that the neutronrich minor isotopes can be measured. A useful feature of the $\mathrm{MgH}$ molecule is that the strength of the $\mathrm{MgH}$ lines does not strongly depend on metallicity. Cottrell (1978) showed that for sufficiently cool stars, a decrease in the metal abundance will weaken the atomic lines, while the $\mathrm{MgH}$ lines remain strong.

The stars listed in Table 1 were observed at McDonald Observatory on the $2.7 \mathrm{~m}$ Harlan J. Smith Telescope between 1999 November and 2002 April. The data were obtained using the cross-dispersed echelle spectrometer (Tull et al. 1995) at the coudé $\mathrm{f} / 32.5$ focus, with a resolving power of $R \equiv \lambda / \Delta \lambda=60,000$ (four stars were observed with a resolving power of about $R=35,000$ ). The detector was a Tektronix CCD, with $24 \mu \mathrm{m}^{2}$ pixels in a $2048 \times 2048$ format. This setting provided spectral coverage from 3800 to $8900 \AA$, with gaps between the orders beyond $5800 \AA$. When necessary, multiple 20-30 minute exposures were coadded to increase the signal-to-noise ratio $(\mathrm{S} / \mathrm{N})$. Although varying from star to star, the typical $\mathrm{S} / \mathrm{N}$ of the extracted one-dimensional spectra was $90 \mathrm{pixel}^{-1}$ at $5140 \AA$. Onedimensional wavelength-calibrated normalized spectra were extracted in the standard way using the IRAF ${ }^{2}$ package of programs.

The stellar parameters and uncertainties were derived in Paper I, and the procedure is repeated briefly here. The

${ }^{2}$ IRAF is distributed by the National Optical Astronomy Observatory, which is operated by the Association of Universities for Research in Astronomy (AURA), Inc., under cooperative agreement with the National Science Foundation. equivalent widths of $\mathrm{Fe}$ I and $\mathrm{Fe}$ II lines were measured using routines in IRAF. The $g f$-values of the lines were taken from Lambert et al. (1996) and a compilation by R. E. Luck (1993, private communication). We adopted NEXTGEN model atmospheres for low-mass stars computed by Hauschildt, Allard, \& Baron (1999), interpolating within the grid when necessary. We made use of the LTE stellar line analysis program MOOG (Sneden 1973) to calculate the abundance of each Fe line based on the measured equivalent width. Effective temperatures $\left(T_{\text {eff }}\right)$ were set from the requirement that the abundances of individual $\mathrm{Fe}$ lines be independent of lower excitation potential. The microturbulence was set by insisting that the abundances of individual Fe lines show no trend against equivalent width. By forcing agreement between the $\mathrm{Fe}$ abundances derived from neutral and ionized lines, the gravity was fixed. This process required iteration until a consistent set of parameters was obtained from which the $\mathrm{Fe}$ abundance was determined from the mean of all $\mathrm{Fe}$ lines. Estimated uncertainties in the model parameters are $\delta T_{\text {eff }}=150 \mathrm{~K}, \delta \log g=0.3$ dex, $\delta \xi_{t}=0.3 \mathrm{~km} \mathrm{~s}^{-1}$, and $\delta[\mathrm{Fe} / \mathrm{H}]=0.2$ dex. We comment below on the influence of these uncertainties on the derived isotopic ratios.

The Galactic space velocities of the sample were also derived in Paper I. In Figure 1 we plot $U$ (positive toward the Galactic center), $V$ (positive in the direction of Galactic rotation), and $W$ (positive toward the north Galactic pole), corrected for the solar motion with respect to the local standard of rest (LSR) versus $[\mathrm{Fe} / \mathrm{H}]$. For the solar motion with respect to the LSR, we adopted the Dehnen \& Binney (1998) values $(+10,+5,+7) \mathrm{km} \mathrm{s}^{-1}$ in $(U, V, W)$. An important point that arises below is that in order to find metalpoor stars, our targets were selected because of their large reduced proper motions, and a majority are kinematically distinct from the thin disk.

A subset of stars listed in Table 1 was not analyzed in Paper I. Seven stars, five giants and two dwarfs, were observed in 2000 August on the $2.7 \mathrm{~m}$ Harlan J. Smith Telescope with a resolving power of $R=120,000$ and a typical S/N of 150 pixel $^{-1}$ at $5140 \AA$. A $20 \AA$ window around $5135 \AA$ was observed. The stellar parameters for these stars

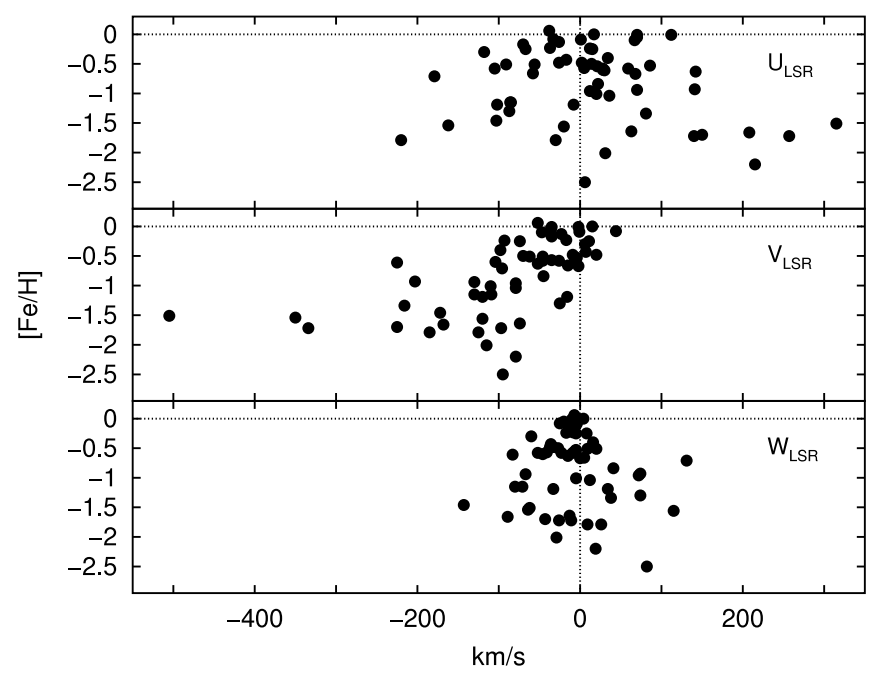

FIG. 1.-Galactic space velocity $U, V$, and $W$ vs. [Fe/H], where $U, V$, and $W$ are relative to the LSR. As expected for a sample selected against the thin disk, a considerable number of stars lag the $\operatorname{LSR}\left(V<-50 \mathrm{~km} \mathrm{~s}^{-1}\right)$. 


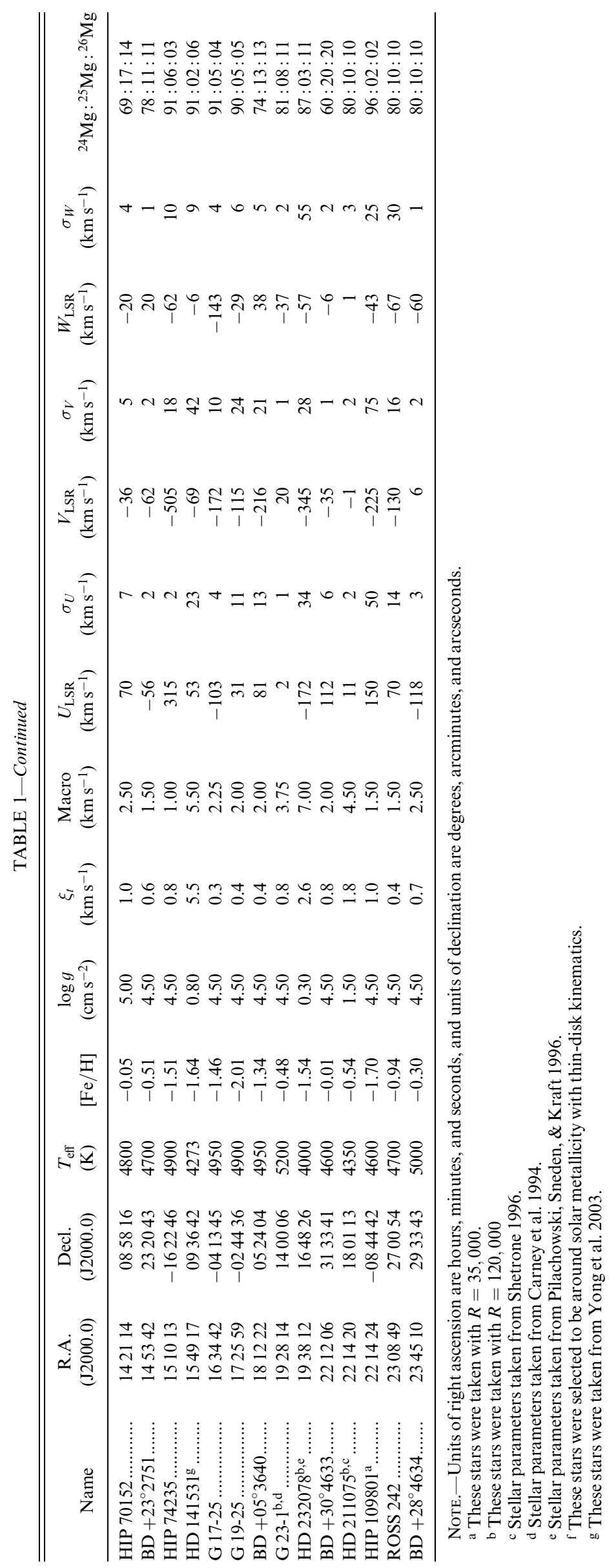


were taken from the literature, as shown in Table 1. A further seven dwarfs were observed with $R=60,000$ and a typical S/N of 200 pixel $^{-1}$ at $5140 \AA$ in 2003 February on the $2.7 \mathrm{~m}$ Harlan J. Smith Telescope. The stellar parameters for these stars were derived in Yong \& Lambert (2003b) using the method outlined in Paper I, also described above. These seven stars were selected to have the kinematics of the thin disk, with metallicities close to the solar value.

\section{ANALYSIS}

The isotopic wavelength splitting in lines from the $\mathrm{MgH}$ $\mathrm{A}-\mathrm{X}$ bands near $5140 \mathrm{~A}$ is small, and so the ${ }^{25} \mathrm{MgH}$ and ${ }^{26} \mathrm{MgH}$ lines are never fully resolved. Instead, ${ }^{25} \mathrm{MgH}$ and ${ }^{26} \mathrm{MgH}$ contribute a red asymmetry to the main ${ }^{24} \mathrm{MgH}$ line. Accordingly, synthetic spectra are generated and fitted to the observed spectrum to derive the isotopic ratio. High resolving powers and high $\mathrm{S} / \mathrm{N}$ ratios are essential for measuring the $\mathrm{Mg}$ isotopic ratios. Our analysis techniques follow the method used by Gay \& Lambert (2000) and McWilliam \& Lambert (1988). Many MgH lines are present in the spectra of cool stars, although few are suitable for isotopic abundance analysis because of blending by identified and unidentified lines. Our $\mathrm{Mg}$ isotopic abundance ratios are derived from three $\mathrm{MgH}$ lines. In Figure 2, we show a region of the spectrum that includes these three $\mathrm{MgH}$ lines, which are a subset of the lines recommended by McWilliam \& Lambert (1988) for extraction of reliable Mg isotopic ratios. These three features are identical to those used by Gay \& Lambert (2000) and are shown in more detail in Figure 3. The feature at $5134.6 \AA$ is a blend of the $Q_{1}(23)$ and $R_{2}(11)$ lines from the $0-0$ band. The red asymmetry on the $\mathrm{MgH}$ features is due to the presence of ${ }^{25} \mathrm{MgH}$ and ${ }^{26} \mathrm{MgH}$. The slightly weaker $\mathrm{MgH}$ features on either side of the 5134.6 A line also exhibit red asymmetric wings, although spectrum synthesis reveals that these lines suffer from contamination, and reliable isotopic ratios cannot be extracted from them (Tomkin \& Lambert 1980). The recommended feature at $5138.7 \AA$ is a blend of the $0-0 Q_{1}(22)$ and $1-1$ $Q_{2}(14) \mathrm{MgH}$ lines. The final recommended feature, at

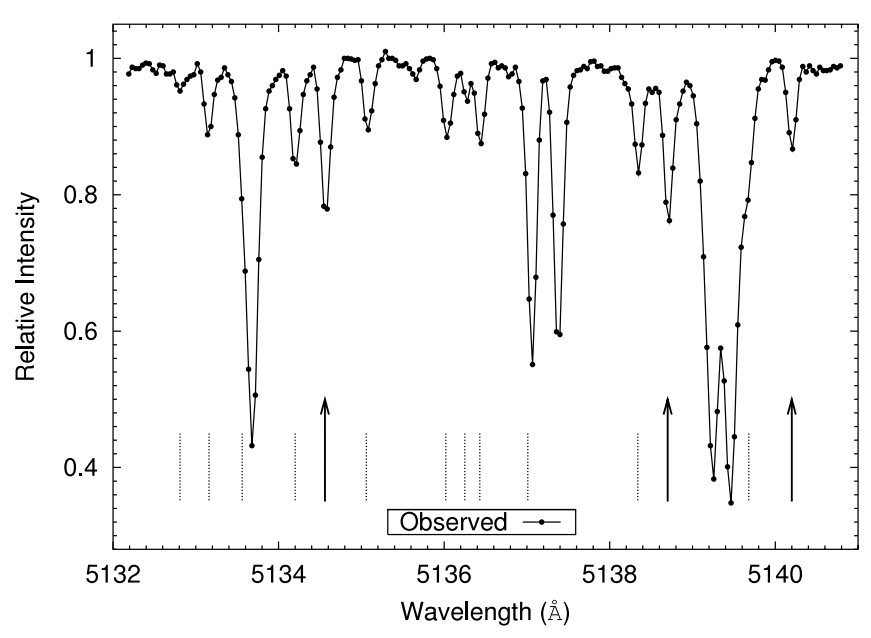

FIG. 2.-Spectrum of G 17-25 from 5132 to $5141 \AA$. The positions of various $\mathrm{MgH}$ A-X $0-0$ and $1-1$ lines are marked below the spectrum. The majority of $\mathrm{MgH}$ lines are unsuitable for isotopic analysis. The positions of the three features that we use to derive the isotopic ratios are highlighted with arrows.
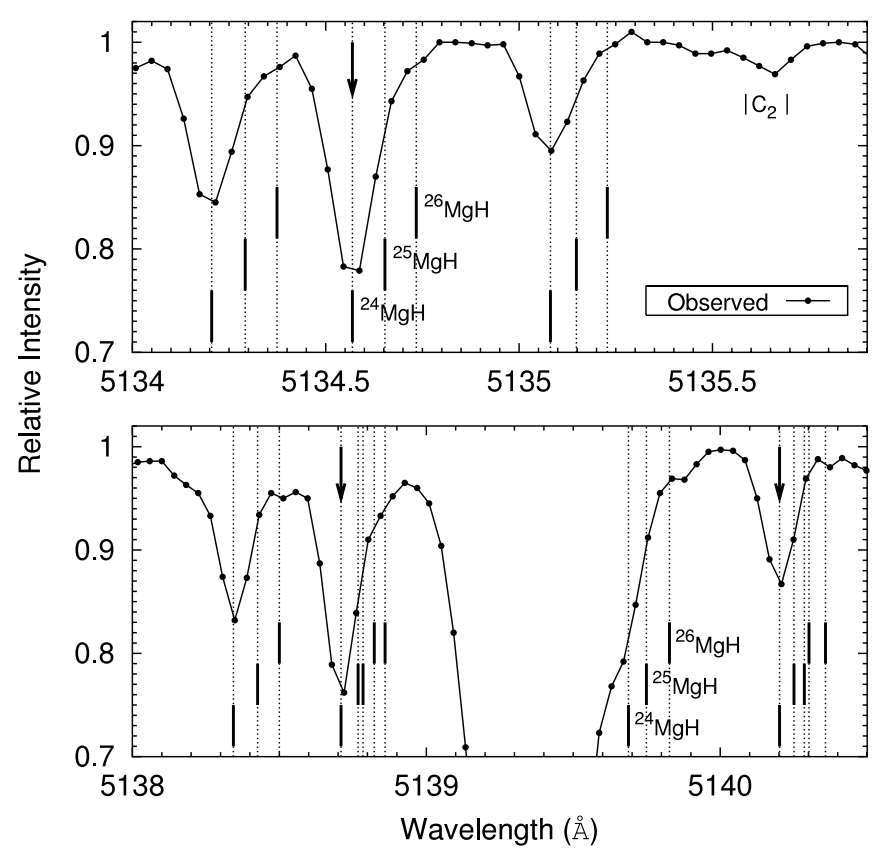

FIG. 3.-Spectrum of G 17-25 from 5134 to $5136 \AA$ (top) and from 5138 to $5140.5 \AA$ (bottom). The positions of the ${ }^{24} \mathrm{MgH},{ }^{25} \mathrm{MgH}$, and ${ }^{26} \mathrm{MgH}$ lines are shown. The lines used in the isotopic analysis to derive the ratios are marked by arrows.

$5140.2 \AA$, is a blend of the $0-0 R_{1}(10)$ and $1-1 R_{2}(4) \mathrm{MgH}$ lines.

To determine the $\mathrm{Mg}$ isotopic abundance ratios, synthetic spectra were produced using MOOG and fitted to the three $\mathrm{MgH}$ features. Our list of atomic and molecular lines was identical to the Gay \& Lambert (2000) list and included contributions from $\mathrm{C}, \mathrm{Mg}, \mathrm{Sc}, \mathrm{Ti}, \mathrm{Cr}, \mathrm{Fe}, \mathrm{Co}, \mathrm{Ni}$, and $\mathrm{Y}$. The wavelengths of all isotopic components were taken from McWilliam \& Lambert (1988) and were based on direct measurements of an $\mathrm{MgH}$ spectrum obtained using a Fourier transform spectrometer by Bernath, Black, \& Brault (1985). The instrumental profile was determined from $\mathrm{Th}$ lines in the spectrum of the Th-Ar comparison lamp. The broadening due to macroturbulence was estimated by fitting the profiles of unblended lines of comparable depth to the $\mathrm{MgH}$ lines. The chosen lines were Ni I at $5115.4 \AA$ and Ti i at $5145.5 \AA$, where typical values for macroturbulence were $1.5-4.0 \mathrm{~km} \mathrm{~s}^{-1}$ (see Fig. 4). These two lines gave the same macroturbulence within $0.25 \mathrm{~km} \mathrm{~s}^{-1}$, and the larger value was adopted if there was a disagreement. Both the macroturbulent and instrumental broadening were assumed to have a Gaussian form. We adjusted the $\mathrm{Mg}$ abundance to best fit the depths of the $\mathrm{MgH}$ lines. The ${ }^{25} \mathrm{Mg}$ and ${ }^{26} \mathrm{Mg}$ abundances were adjusted by trial and error until the profile of a recommended feature was fitted. We did not require the abundances of ${ }^{25} \mathrm{Mg}$ and ${ }^{26} \mathrm{Mg}$ to be equal. For a given star, the final isotopic ratio of ${ }^{24} \mathrm{Mg}:{ }^{25} \mathrm{Mg}:{ }^{26} \mathrm{Mg}$ was the value that provided the best fit to all three recommended features. The best fit was determined by eye, and the differences between the observed spectra and the best-fitting syntheses were similar for all stars. The derived $\mathrm{Mg}$ isotopic ratios are presented in Table 1.

In Figure 5, we compare the observed and synthetic spectra for $\mathrm{G} 9-13$, a subdwarf with $[\mathrm{Fe} / \mathrm{H}]=-0.58$. The strength of the $\mathrm{MgH}$ features is comparable to the strength 

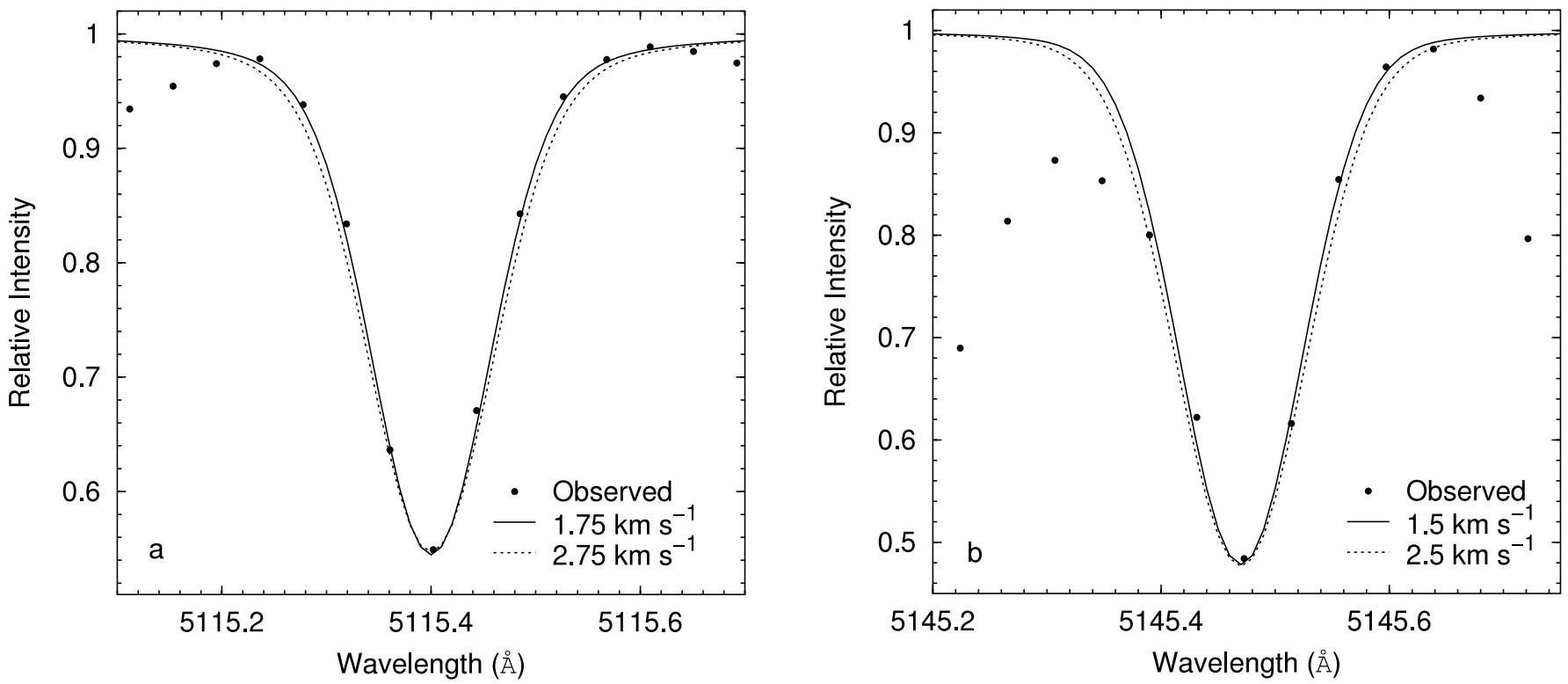

FIG. 4.- Spectrum of G 9-13, showing the lines of Ni I at $5115.4 \AA$ (left) and Ti I at $5145.5 \AA$ (right) from which the macroturbulent broadening is determined.

of the lines from which we derived the macroturbulence (compare Figs. 4 and 5). The red asymmetry of the $\mathrm{MgH}$ lines demands positive contributions from the ${ }^{25} \mathrm{Mg}$ and ${ }^{26} \mathrm{Mg}$ isotopes. The $\mathrm{Mg}$ isotopic ratio ${ }^{24} \mathrm{Mg}:{ }^{25} \mathrm{Mg}:{ }^{26} \mathrm{Mg}=$ $83: 8: 9$ provides an excellent fit to the three recommended $\mathrm{MgH}$ features. Note the poor fit of the pure ${ }^{24} \mathrm{Mg}$ mix $\left({ }^{24} \mathrm{Mg}:{ }^{25} \mathrm{Mg}:{ }^{26} \mathrm{Mg}=100: 0: 0\right)$ to all the $\mathrm{MgH}$ lines. Unsatisfactory ratios $77: 11: 12$ and $89: 5: 6$ are overplotted to give an indication of the measurement uncertainties.

In Figure 6, we show the comparison of the observed and synthetic spectra for LHS 2715, a subdwarf with

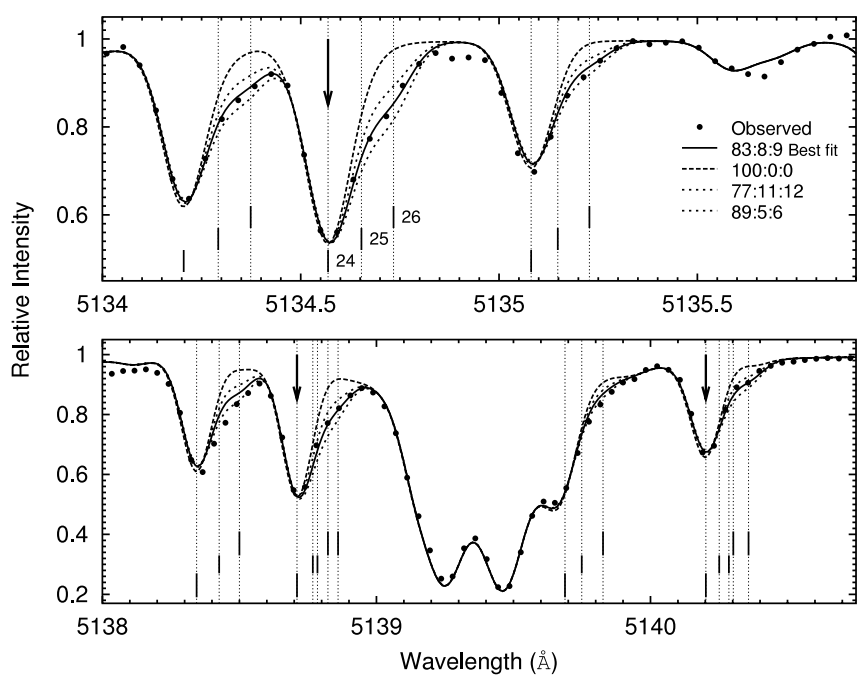

FIG. 5.- Spectrum of G 9-13 from 5134 to $5136 \AA$ (top) and from 5138 to $5140.5 \AA$ (bottom). The features we are interested in fitting are marked from above by arrows. The positions of the ${ }^{24} \mathrm{MgH},{ }^{25} \mathrm{MgH}$, and ${ }^{26} \mathrm{MgH}$ lines are indicated by vertical dotted lines. The circles represent the observed spectrum. The best fit to the recommended features is shown as a solid line for ${ }^{24} \mathrm{Mg}:{ }^{25} \mathrm{Mg}:{ }^{26} \mathrm{Mg}=83: 8: 9$. The pure ${ }^{24} \mathrm{Mg}$ synthesis $(100: 0: 0)$ is plotted as a dashed line and clearly provides a poor fit. The dotted lines represent unsatisfactory ratios $77: 11: 12$ and $89: 5: 6$.
$[\mathrm{Fe} / \mathrm{H}]=-1.56$. The $\mathrm{MgH}$ lines are very strong in this star. The best-fitting $\mathrm{Mg}$ isotopic ratio is $88: 7: 5$. This ratio provides an excellent fit to all three recommended features. Note again the poor fit of the pure ${ }^{24} \mathrm{Mg}$ mix to all the $\mathrm{MgH}$ lines. Unsatisfactory ratios $82: 10: 8$ and $97: 1: 2$ are overplotted to give an indication of the measurement uncertainties. Other $\mathrm{MgH}$ lines adjacent to the recommended features show stronger red asymmetries, indicating the presence of unidentified blends.

In Figure 7, we plot observed and synthetic spectra for PLX 5805, a subdwarf with $[\mathrm{Fe} / \mathrm{H}]=-1.72$. This star also has strong $\mathrm{MgH}$ lines, although not quite as strong as those seen in LHS 2715. This difference is likely due to the $T_{\text {eff }}$ difference rather than the difference in metallicity. The

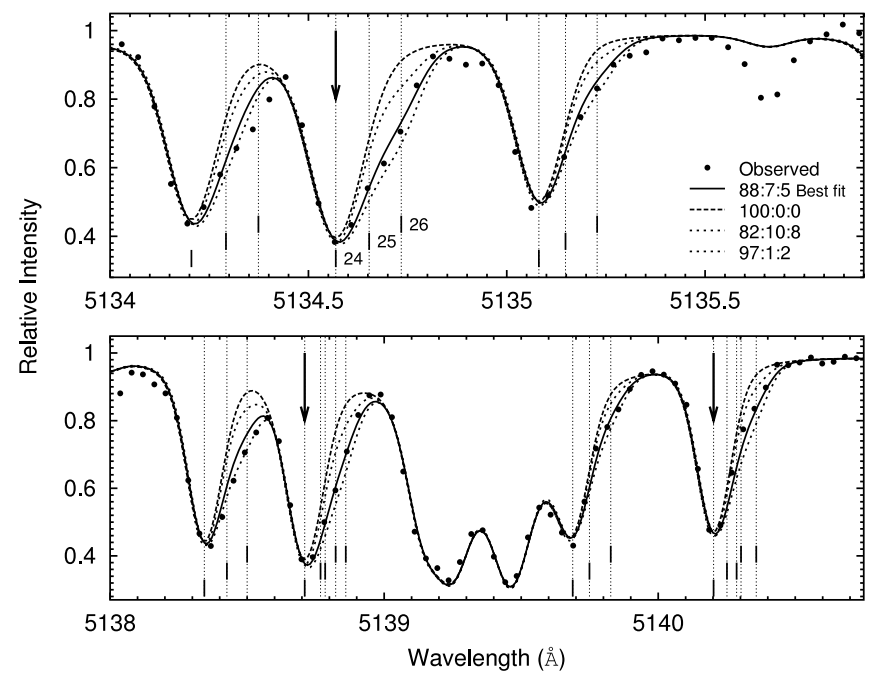

FIG. 6.- Spectrum of LHS 2715 from 5134 to $5136 \AA$ (top) and from 5138 to $5140.5 \AA$ (bottom). The best fit to the recommended features is shown as a solid line for ${ }^{24} \mathrm{Mg}:{ }^{25} \mathrm{Mg}:{ }^{26} \mathrm{Mg}=88: 7: 5$. The pure ${ }^{24} \mathrm{Mg}$ synthesis $(100: 0: 0)$ is plotted as a dashed line and clearly provides a poor fit. The dotted lines represent unsatisfactory ratios $82: 10: 8$ and $97: 1: 2$. 


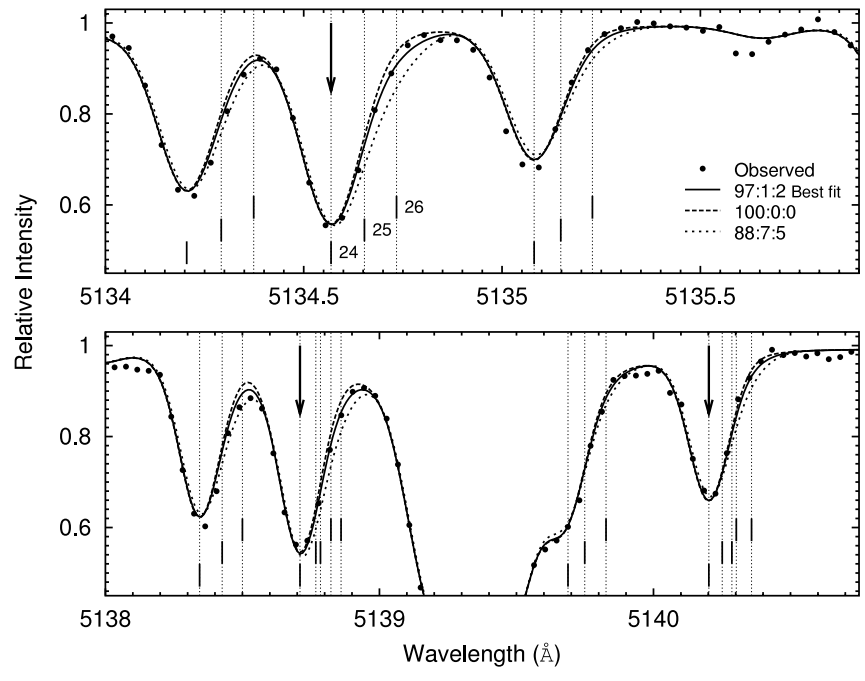

Fig. 7.-Spectrum of PLX 5805 from 5134 to $5136 \AA$ (top) and from 5138 to $5140.5 \AA$ (bottom). The best fit to the recommended features is shown as a solid line for ${ }^{24} \mathrm{Mg}:{ }^{25} \mathrm{Mg}:{ }^{26} \mathrm{Mg}=97: 1: 2$. The pure ${ }^{24} \mathrm{Mg}$ synthesis $(100: 0: 0)$ is plotted as a dashed line. The dotted line represent $88: 7: 5$, which is clearly a poor fit to the data.

best-fitting isotopic ratio is $97: 1: 2$, which again provides a good fit to all three recommended features. Within the uncertainties, the pure ${ }^{24} \mathrm{Mg}$ synthesis also provides a reasonable fit to the data. An unsatisfactory ratio $88: 7: 5$, the isotopic mix that fits LHS 2715, is overplotted to highlight the measurement uncertainties. (This star was observed at $R=35,000$.)

In the fitting of synthetic spectra to observed line profiles, sources of error include continuum fitting, microturbulence, macroturbulence, and identified and unidentified blends. To understand the effects of these errors, we generated a synthetic spectrum, assuming $T_{\text {eff }}=4300 \mathrm{~K}, \log g=4.5$, $[\mathrm{Fe} / \mathrm{H}]=-2.5, \xi_{t}=0.5 \mathrm{~km} \mathrm{~s}^{-1}$, macroturbulence of $2.0 \mathrm{~km}$ $\mathrm{s}^{-1},{ }^{24} \mathrm{Mg}:{ }^{25} \mathrm{Mg}:{ }^{26} \mathrm{Mg}=94: 3: 3$, and $R=60,000$. We added noise to the synthesis to produce a spectrum with $\mathrm{S} / \mathrm{N}=100$ pixel $^{-1}$. We then treated this artificial spectrum as real data. Assuming the correct input parameters ( $T_{\text {eff }}$, $\log g, \xi_{t}$, macroturbulence, and $[\mathrm{Fe} / \mathrm{H}]$ ), our best-fitting ratio to the artificial spectrum was $96: 2: 2$. Incorrect choices for $T_{\text {eff, }}$ gravity, or metallicity equally affect the ${ }^{24} \mathrm{MgH},{ }^{25} \mathrm{MgH}$, and ${ }^{26} \mathrm{MgH}$ lines, and so the measured isotopic ratios are quite insensitive to the adopted model parameters. For strong lines, the microturbulence would affect the derived isotopic ratio, as the strong ${ }^{24} \mathrm{MgH}$ line is more sensitive to the adopted microturbulence than the weaker ${ }^{25} \mathrm{MgH}$ and ${ }^{26} \mathrm{MgH}$ lines. We increased the microturbulence by $0.5 \mathrm{~km} \mathrm{~s}^{-1}$ and measured $97: 1: 2$. We decreased the continuum by $0.5 \%$ and measured $96: 2: 2$. We increased the macroturbulence by $0.5 \mathrm{~km} \mathrm{~s}^{-1}$ and measured $97: 2: 1$. An identical test was performed on a synthetic spectrum generated assuming $T_{\text {eff }}=4800 \mathrm{~K}$, $\log g=4.5, \quad[\mathrm{Fe} / \mathrm{H}]=-0.25, \quad \xi_{t}=0.6 \quad \mathrm{~km} \mathrm{~s}^{-1}$, macroturbulence of $2.5 \mathrm{~km} \mathrm{~s}^{-1},{ }^{24} \mathrm{Mg}:{ }^{25} \mathrm{Mg}:{ }^{26} \mathrm{Mg}=80: 10: 10$, $R=60,000$, and $\mathrm{S} / \mathrm{N}=90 \mathrm{pixel}^{-1}$. In both tests we found that errors in the model parameters ( $T_{\text {eff }}, \log g, \xi_{t}$, macroturbulence, and continuum) are about $b \pm 1$ and $c \pm 1$ when the ratios are expressed as ${ }^{24} \mathrm{Mg}:{ }^{25} \mathrm{Mg}:{ }^{26} \mathrm{Mg}=$ $(100-b-c): b: c$. Inspection of Figures 5-7 suggests that the uncertainties in determining the best fit are at the
TABLE 2

Comparison of the Mg Isotopic Ratios With the GAY \& LAMBERT 2000 VALUes

\begin{tabular}{|c|c|c|c|}
\hline \multirow[b]{2}{*}{ ОВЈеСт } & \multirow[b]{2}{*}{ OTHER NAME } & \multicolumn{2}{|c|}{${ }^{24} \mathrm{Mg}:{ }^{25} \mathrm{Mg}:{ }^{26} \mathrm{Mg}$} \\
\hline & & This Study & Gay \& Lambert 2000 \\
\hline GJ 1064A ........... & HD 23439A & $80: 14: 06$ & $78: 13: 09$ \\
\hline GJ 1064B .......... & HD 23439B & $84: 08: 08$ & $84: 08: 08$ \\
\hline GL $158 \ldots \ldots \ldots \ldots \ldots$ & HD 25329 & $84: 08: 08$ & $85: 08: 08$ \\
\hline G $87-27 \ldots \ldots \ldots \ldots$ & $\mathrm{BD}+37^{\circ} 1665$ & $82: 11: 07$ & $85: 09: 06$ \\
\hline HD $114095 \ldots \ldots .$. & & $83: 08: 08$ & $79: 13: 08$ \\
\hline HIP $74235 \ldots \ldots \ldots .$. & HD 134439 & $91: 06: 03$ & $91: 06: 03$ \\
\hline
\end{tabular}

Note.-There is a good agreement particularly for the ratio ${ }^{26} \mathrm{Mg} /{ }^{24} \mathrm{Mg}$.

level $b \pm 3$ and $c \pm 3$ when the ratios are expressed as ${ }^{24} \mathrm{Mg}:{ }^{25} \mathrm{Mg}:{ }^{26} \mathrm{Mg}=(100-b-c): b: c$. The ratio ${ }^{25} \mathrm{Mg} /{ }^{24} \mathrm{Mg}$ is less accurately determined than the ${ }^{26} \mathrm{Mg} /{ }^{24} \mathrm{Mg}$ ratio because of the larger isotopic shift of ${ }^{26} \mathrm{MgH}$. That is, ${ }^{26} \mathrm{MgH}$ is less blended with the strong ${ }^{24} \mathrm{MgH}$ line than ${ }^{25} \mathrm{MgH}$.

There are six stars in common with the Gay \& Lambert (2000) sample, almost a third of their sample. In Table 2, we compare the $\mathrm{Mg}$ isotopic ratios derived in the two different studies. Since the analysis techniques are essentially identical, the differences between the two studies can be attributed mainly to the quality of the data. The Gay \& Lambert data are superior in both resolution $(R=150,000$ vs. $R=60,000)$ and $\mathrm{S} / \mathrm{N}\left(150\right.$ vs. 90 pixel $\left.^{-1}\right)$. The agreement is excellent for the six stars common to both samples, particularly for the ratio ${ }^{26} \mathrm{Mg} /{ }^{24} \mathrm{Mg}$. Gay \& Lambert state that their errors are around $b \pm 2$ and $c \pm 2$ when the ratios are expressed as ${ }^{24} \mathrm{Mg}:{ }^{25} \mathrm{Mg}:{ }^{26} \mathrm{Mg}=(100-b-c): b: c$. That is, our estimated errors are $50 \%$ larger than those reported by Gay \& Lambert, which reflects the difference in data quality.

In Table 3, we compare five $\mathrm{Mg}$ isotopic ratios measured in giants with those reported by Shetrone (1996). Shetrone's data were taken at $R=60,000$, with an $\mathrm{S} / \mathrm{N}$ ranging from about 80 to 160 per resolution element. Our data were taken at $R=120,000$, with an $\mathrm{S} / \mathrm{N}$ of around 150 pixel $^{-1}(210$ per resolution element). More importantly, the Shetrone study employed a different set of $\mathrm{MgH}$ lines to derive the isotopic ratios. Although Shetrone could not distinguish the contribution of ${ }^{25} \mathrm{Mg}$ from that of ${ }^{26} \mathrm{Mg}$, we find a fair agreement

TABLE 3

Comparison of the Mg Isotopic Ratios with the Shetrone 1996 Values

\begin{tabular}{|c|c|c|}
\hline \multirow[b]{2}{*}{ ОвЈеСт } & \multicolumn{2}{|c|}{${ }^{24} \mathrm{Mg}:{ }^{25} \mathrm{Mg}:{ }^{26} \mathrm{Mg}$} \\
\hline & This Study & Shetrone 1996 \\
\hline HD $5098 \ldots \ldots \ldots \ldots \ldots$ & $80: 08: 12$ & $90: 05: 05$ \\
\hline HD $9731 \ldots \ldots \ldots \ldots \ldots$ & $75: 12: 13$ & $80: 10: 10$ \\
\hline HD $211075 \ldots \ldots \ldots \ldots . . . . . .$. & $80: 10: 10$ & $92: 04: 04$ \\
\hline HD $103036 \ldots \ldots \ldots \ldots . . . .$. & $94: 00: 06^{\mathrm{a}}$ & $94: 03: 03$ \\
\hline HD $141531 \ldots \ldots \ldots \ldots . . . .$. & $91: 02: 06^{\mathrm{a}}$ & $90: 05: 05$ \\
\hline
\end{tabular}

a $\mathrm{Mg}$ isotopic ratio from Yong et al. 2003, based on high-resolution $(R=110,000)$, high-S/N $\left(250\right.$ pixel $\left.^{-1}\right)$ spectra obtained with UVES on the Very Large Telescope. 
for three stars. For the other two stars, we find a poor agreement with Shetrone's values because of the different data quality and choice of $\mathrm{MgH}$ lines.

\section{DISCUSSION}

\subsection{Observed Trends}

The evolution of the $\mathrm{Mg}$ isotopic ratios with metallicity is shown in Figures 8,9 , and 10 . The ratios ${ }^{25} \mathrm{Mg} /{ }^{24} \mathrm{Mg}$, ${ }^{26} \mathrm{Mg} /{ }^{24} \mathrm{Mg}$, and ${ }^{26} \mathrm{Mg} /{ }^{25} \mathrm{Mg}$ are plotted against $[\mathrm{Fe} / \mathrm{H}]$. We combine our sample with data from the Gay \& Lambert (2000) study, in which the total sample consists of 75 different stars. The observed ratios ${ }^{25} \mathrm{Mg} /{ }^{24} \mathrm{Mg}$ and ${ }^{26} \mathrm{Mg} /{ }^{24} \mathrm{Mg}$ decline with decreasing metallicity. Below $[\mathrm{Fe} / \mathrm{H}]=-1.5$, there are six stars that show nonzero ratios, ${ }^{26} \mathrm{Mg} /{ }^{24} \mathrm{Mg}>0.05$. Even taking into account the errors, it is unlikely that these six stars have ${ }^{26} \mathrm{Mg} /{ }^{24} \mathrm{Mg}=0$. Around solar metallicity, the solar ratios ${ }^{25} \mathrm{Mg} /{ }^{24} \mathrm{Mg}$ and ${ }^{26} \mathrm{Mg} /{ }^{24} \mathrm{Mg}$ lie at the lower boundary. The ratio ${ }^{26} \mathrm{Mg} /{ }^{25} \mathrm{Mg}$ is essentially constant and centered at unity. The solar ratio ${ }^{26} \mathrm{Mg} /{ }^{25} \mathrm{Mg}$ does not appear to be unusual. In Figure 10, there are three stars with large excesses of ${ }^{26} \mathrm{Mg}$, or equivalently, underabundances of ${ }^{25} \mathrm{Mg}$. These three stars have ratios of $87: 3: 11,91: 2: 6$, and $97: 1: 2$, and within the measurement uncertainties $\left( \pm 3\right.$ for both ${ }^{25} \mathrm{Mg}$ and ${ }^{26} \mathrm{Mg}$ ), all stars could have ${ }^{25} \mathrm{Mg} \simeq{ }^{26} \mathrm{Mg}$.

Gay \& Lambert showed that at a given $[\mathrm{Fe} / \mathrm{H}]$, there was a scatter in the isotopic ratios exceeding the measurement errors. For our sample, we confirm the spread in ${ }^{25} \mathrm{Mg} /{ }^{24} \mathrm{Mg}$ and ${ }^{26} \mathrm{Mg} /{ }^{24} \mathrm{Mg}$, and the dispersion appears to increase with increasing metallicity. Figures 8 and 9 show a real scatter at low metallicity. In $\S 3$, we plotted observed and synthetic spectra for two stars with similar metallicities and atmospheric parameters, LHS $2715([\mathrm{Fe} / \mathrm{H}]=-1.56)$ and PLX $5805([\mathrm{Fe} / \mathrm{H}]=-1.72)$. The best-fitting ratio to LHS 2715 is $88: 7: 5$, and the best-fitting ratio to PLX 5805 is $97: 1: 2$. In Figure 6, we overplot the ratio $97: 1: 2$ on the spectrum of LHS 2715 to show that it provides a poor fit. Likewise, in Figure 7, we overplot the ratio $88: 7: 5$ on the spectrum of PLX 5805 and find that it provides a poor fit. At higher metallicity, Figure 11 shows that the scatter certainly exceeds the measurement errors. In this figure, we

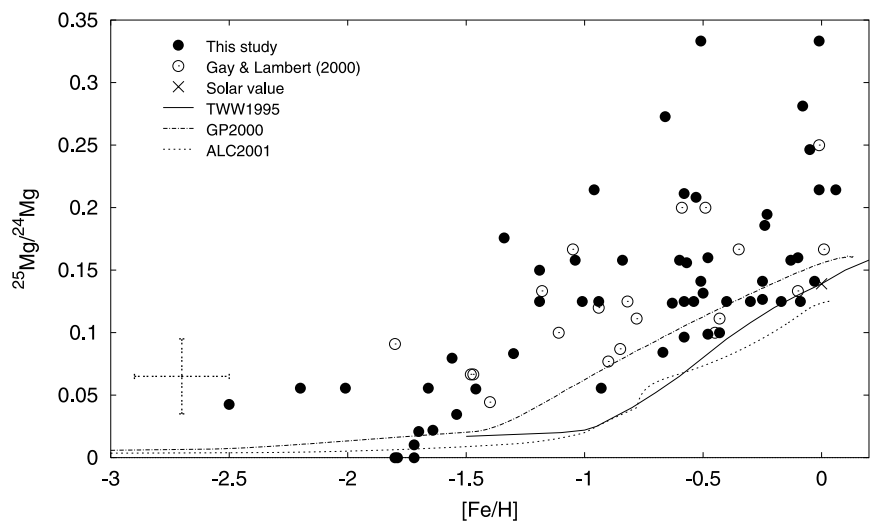

FIG. 8.-Evolution of isotopic ratio ${ }^{25} \mathrm{Mg} /{ }^{24} \mathrm{Mg}$ vs. $[\mathrm{Fe} / \mathrm{H}]$. The filled circles represent data from this study, the open circles represent the Gay \& Lambert (2000) data, and the cross marks the solar value. (The Gay \& Lambert value is plotted for the stars in common.) A representative error bar is given. The three lines are for the TWW1995, GP2000, and ALC2001 predictions.

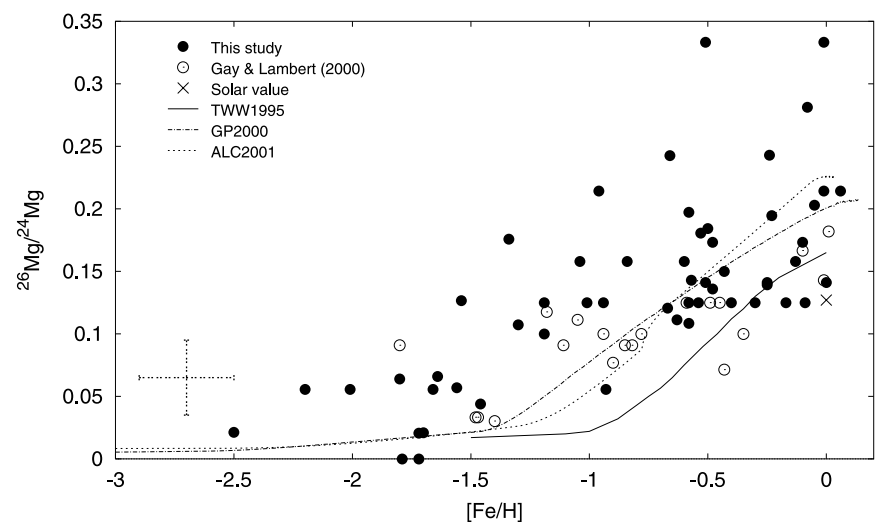

FIG. 9.- Same as Fig. 8, but for ${ }^{26} \mathrm{Mg} /{ }^{24} \mathrm{Mg}$

plot the observed spectra of two stars, BD $+04^{\circ} 415$ $([\mathrm{Fe} / \mathrm{H}]=-0.63)$ and LP 734-54 $([\mathrm{Fe} / \mathrm{H}]=-0.66)$, with similar metallicities and almost identical atmospheric parameters. The measured isotopic ratios are $81: 10: 9$ for BD $+04^{\circ} 415$ and $66: 18: 16$ for LP $734-54$. The best ratio for BD $+04^{\circ} 415$ provides a very poor fit when compared with the observed spectrum of LP 734-54. Similarly, the isotopic ratio offering the best fit to LP 734-54 provides a poor fit to the observed spectrum of $\mathrm{BD}+04^{\circ} 415$. In short, there is a real scatter in the measured $\mathrm{Mg}$ isotope ratios at low and high metallicities.

For a given metallicity, our stars generally exhibit higher isotopic ratios $\left({ }^{25} \mathrm{Mg} /{ }^{24} \mathrm{Mg}\right.$ and ${ }^{26} \mathrm{Mg} /{ }^{24} \mathrm{Mg}$ ) than those within the Gay \& Lambert sample. This is particularly true at the higher metallicities, $[\mathrm{Fe} / \mathrm{H}]>-1.0$. As mentioned above, Table 2 shows the Mg isotopic ratios for six stars common to both studies for which the agreement is excellent. This agreement suggests that the high values of ${ }^{25} \mathrm{Mg} /{ }^{24} \mathrm{Mg}$ and ${ }^{26} \mathrm{Mg} /{ }^{24} \mathrm{Mg}$ are real.

BD $+30^{\circ} 4633$ and LP $790-19$ both show large ratios $(\simeq 0.3)$ for both ${ }^{25} \mathrm{Mg} /{ }^{24} \mathrm{Mg}$ and ${ }^{26} \mathrm{Mg} /{ }^{24} \mathrm{Mg}$. The $\mathrm{MgH}$ lines in these stars are rather strong. Since these unusually large isotopic ratios were found in stars with strong $\mathrm{MgH}$ lines, we conducted an additional test to determine if there were systematic errors in the analysis. We changed the macroturbulence and microturbulence by $0.5 \mathrm{~km} \mathrm{~s}^{-1}$. Assuming these new values, we remeasured the $\mathrm{Mg}$ isotope ratios. In both cases, the measured $\mathrm{Mg}$ isotope ratio changed by only $b \pm 2$ and $c \pm 2$ when the ratio is expressed as

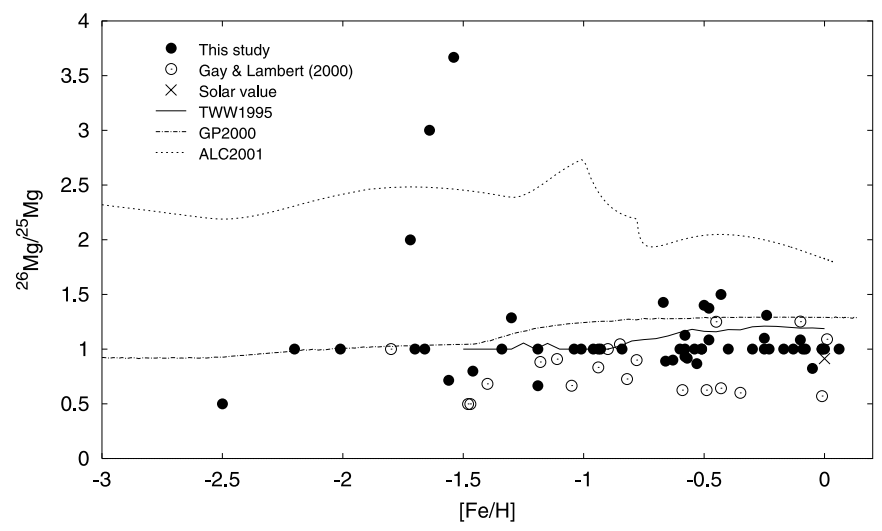

FIG. 10. - Same as Fig. 8, but for ${ }^{26} \mathrm{Mg} /{ }^{25} \mathrm{Mg}$ 


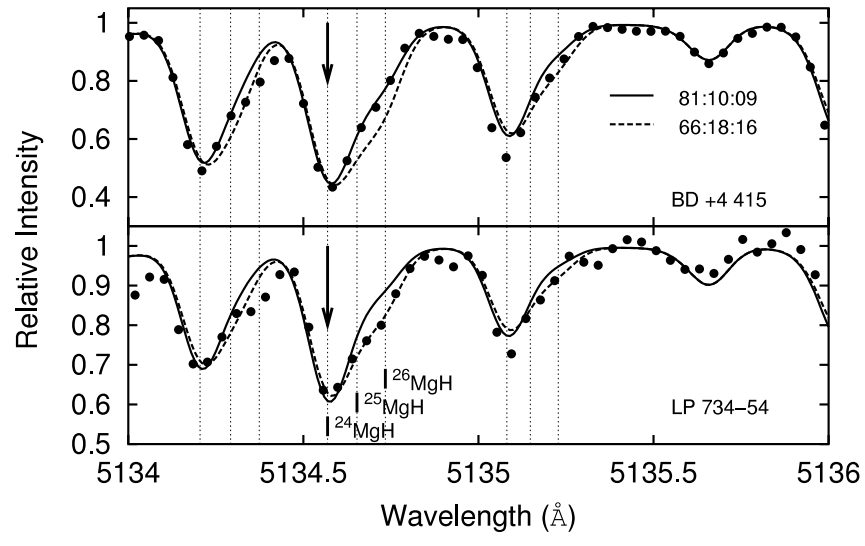

FIG. 11.-Spectra of BD $+04^{\circ} 415$ (top) and LP 734-54 (bottom). In both panels, the observed spectra are shown as circles and two different isotopic ratios are plotted, 81:10:9 (solid line) and 68:18:16 (dashed line). The ratio $81: 10: 9$ is the best fit for $\mathrm{BD}+04^{\circ} 415$, while $68: 18: 16$ is the best fit for LP 734-54. The positions of the ${ }^{24} \mathrm{MgH},{ }^{25} \mathrm{MgH}$, and ${ }^{26} \mathrm{MgH}$ lines are indicated. The red asymmetries are very different between these two stars, and the difference in the $\mathrm{Mg}$ isotopic ratios exceeds the measurement errors.

${ }^{24} \mathrm{Mg}:{ }^{25} \mathrm{Mg}:{ }^{26} \mathrm{Mg}=(100-b-c): b: c$. As discussed above, the analysis techniques provide firm limits on the error bars. Giants in metal-poor globular clusters have shown $\left({ }^{25} \mathrm{Mg}+{ }^{26} \mathrm{Mg}\right) /{ }^{24} \mathrm{Mg} \simeq 1.0$ (Shetrone 1996) and ${ }^{24} \mathrm{Mg}:{ }^{25} \mathrm{Mg}:{ }^{26} \mathrm{Mg}=53: 9: 39$ (Yong et al. 2003), values comparable to and greater than the highest isotopic ratios found in this study.

While there is no obvious trend of ${ }^{25} \mathrm{Mg} /{ }^{24} \mathrm{Mg}$ and ${ }^{26} \mathrm{Mg} /{ }^{24} \mathrm{Mg}$ with $T_{\text {eff }}$, it is curious that the two stars with ${ }^{25} \mathrm{Mg} /{ }^{24} \mathrm{Mg}$ and ${ }^{26} \mathrm{Mg} /{ }^{24} \mathrm{Mg}$ of $\simeq 0.3$ are both cool and have

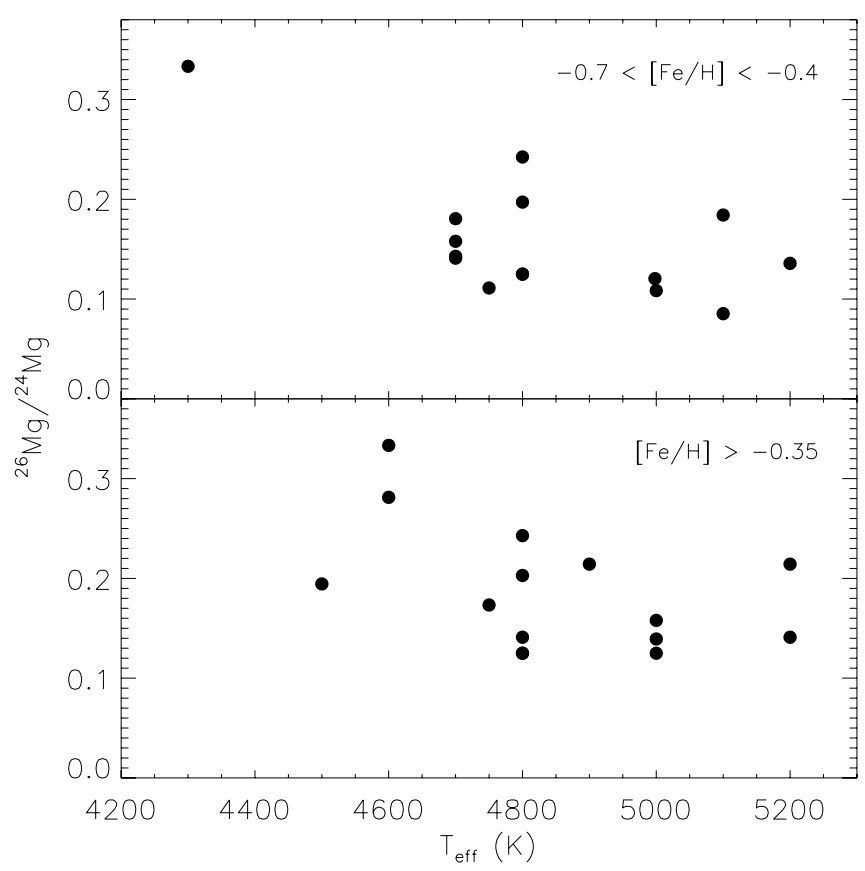

FIG. 12.- Mg isotope ratio ${ }^{26} \mathrm{Mg} /{ }^{24} \mathrm{Mg}$ vs. $T_{\text {eff }}$ for two $[\mathrm{Fe} / \mathrm{H}]$ intervals. In the top panel, the data cover $-0.7<[\mathrm{Fe} / \mathrm{H}]<-0.4$, and in the bottom panel, the data cover $[\mathrm{Fe} / \mathrm{H}]>-0.35$. The ratio ${ }^{26} \mathrm{Mg} /{ }^{24} \mathrm{Mg}$ may increase with decreasing $T_{\text {eff }}$ strong $\mathrm{MgH}$ lines. In Figure 12, we show the ${ }^{26} \mathrm{Mg} /{ }^{24} \mathrm{Mg}$ ratio for dwarfs versus $T_{\text {eff }}$ for two $[\mathrm{Fe} / \mathrm{H}]$ intervals. There is a hint that the isotopic ratio increases with decreasing $T_{\text {eff. If }}$ confirmed by further exploration of cool dwarfs, it may point to an inadequacy of the classical model atmospheres and/or the assumption of LTE for $\mathrm{MgH}$ line formation.

An assumption of classical atmospheres is that of homogeneous layers. Suppose the real atmosphere consists of cool and hot columns, with $\mathrm{MgH}$ lines represented strongly in the spectrum of the former but more weakly in the latter. The continuum from the hot columns dilutes the $\mathrm{MgH}$ lines from the cool columns. In analyzing the combined spectrum of the cool and hot columns with a classical atmosphere, one underestimates the level of saturation of the $\mathrm{MgH}$ lines and overestimates the ${ }^{25} \mathrm{Mg} /{ }^{24} \mathrm{Mg}$ and ${ }^{26} \mathrm{Mg} /{ }^{24} \mathrm{Mg}$ ratios for those stars in which the $\mathrm{MgH}$ lines are strong. Just such an effect was suggested by Lambert, Mallia, \& Petford (1971) to account for a report of high (nonterrestrial) isotopic ratios from spectra of sunspot umbrae; the bright umbral dots seen in high-resolution images of sunspots serve as the hot columns in this example. Velocity differences between cool and hot columns and within a column would be an additional factor not included in our analysis, but the isotopic shifts are several times the expected Doppler shifts arising from stellar granulation. A thorough analysis of $\mathrm{MgH}$ (and other) lines from the weakest to the strongest might shed light on the extent of the inhomogeneities. It should be noted that stars of very similar atmospheric parameters may show quite different isotopic ratios, suggesting that, if a failure of classical atmospheres is the responsible factor, the inhomogeneities are not simply dependent on effective temperature, surface gravity, and metallicity.

Departures from LTE could lead to systematic errors in the isotopic ratios. Suppose that the line source function exceeds everywhere the local Planck function. Then, the ratio of the ${ }^{25} \mathrm{MgH}$ and ${ }^{26} \mathrm{MgH}$ line to the ${ }^{24} \mathrm{MgH}$ line will be less than expected in LTE. An LTE analysis of these lines will likely result in a systematic overestimate of the isotopic ratios. The fact that the scatter in isotopic ratios among the stars is present for almost identical stars, independent of effective temperature, and present in dwarfs and giants suggests that departures from LTE are not a major influence on the derived ratios.

\subsection{Model Predictions}

Three different models predict the evolution of the elements from carbon through zinc, including the $\mathrm{Mg}$ isotopes: TWW1995, GP2000, and ALC2001. Underpinning each of these models are assumptions regarding the dynamical evolution of the Galaxy, the IMF, the star formation rate, and the stellar yields. We briefly compare and contrast various assumptions between the three different models, with an emphasis on those that would affect the predicted evolution of the $\mathrm{Mg}$ isotopic ratios. Of the major ingredients, only the stellar yields can be calculated from first principles, although particular reaction rates may be plagued by uncertainties. For massive stars that die as Type II supernovae, all three models rely on the Woosley \& Weaver (1995) yields that include all isotopes of $\mathrm{Mg}$, along with Fe. TWW1995 also consider the contribution from intermediate-to-lowmass stars that become planetary nebulae and intermediateto-low-mass stars that become Type Ia supernovae. 
TWW1995 assumed a Salpeter IMF and a dynamical model for the Galactic disk. The GP2000 study differs from TWW1995 by using "appropriate models for both the halo and disk," along with the Kroupa, Tout, \& Gilmore (1993) IMF, "which presumably describes the distribution of stellar masses better than the Salpeter IMF." GP2000 deliberately neglect the contribution from intermediate-mass stars, in order to gauge " to what extent those stars (or other sources) are required to account for the observations." ALC2001 also treat the halo and disk independently and use " metallicity-dependent stellar yields for the whole range of stellar masses considered." ALC2001 also adopt the Kroupa et al. (1993) IMF. For all three models, Type II and Type Ia supernovae are responsible for the production of Fe, whereas Type II supernovae are responsible for the production of the Mg isotopes. The TWW1995 predictions offer a reasonable fit to observed elemental abundances (e.g., $\mathrm{Mg}, \mathrm{Si}$, and $\mathrm{Ca}$ ), provided that the $\mathrm{Fe}$ yields from Type II supernovae are reduced by a factor of 2 . The observed elemental abundances (e.g., $\mathrm{Mg}, \mathrm{Si}$, and $\mathrm{Ca}$ ) are reproduced by both the GP2000 and ALC2001 predictions.

In Figures 8-10, we overplot the TWW1995, GP2000, and ALC2001 predictions, along with our measured Mg isotopic ratios and the Gay \& Lambert (2000) measurements. The three predictions are qualitatively similar, which is unsurprising, as all three models rely on the same source for yields from massive stars. The abundances of ${ }^{25} \mathrm{Mg}$ and ${ }^{26} \mathrm{Mg}$ with respect to ${ }^{24} \mathrm{Mg}$ fall away with decreasing metallicity. This decrease in ${ }^{25} \mathrm{Mg} /{ }^{24} \mathrm{Mg}$ and ${ }^{26} \mathrm{Mg} /{ }^{24} \mathrm{Mg}$ is due to the decrease in the abundance of ${ }^{22} \mathrm{Ne}$, the seed from which the heavy isotopes are produced via ${ }^{22} \mathrm{Ne}(\alpha, n)^{25} \mathrm{Mg}(n$, $\gamma)^{26} \mathrm{Mg}$. The predicted $\mathrm{Mg}$ isotopic ratios suggest that once the metallicity in massive stars reaches a critical value, the production of ${ }^{25} \mathrm{Mg}$ and ${ }^{26} \mathrm{Mg}$ becomes increasingly efficient. This is consistent with the Woosley \& Weaver (1995) massive-star yields. At low metallicities, the nonzero plateau in the predicted isotopic ratios reflects the primary production of the neutron-rich $\mathrm{Mg}$ isotopes. Primary production takes place because massive, metal-poor stars produce $\mathrm{C}$, $\mathrm{N}$, and $\mathrm{O}$, which can then be burned into ${ }^{22 \mathrm{Ne}}$. The abundances of ${ }^{26} \mathrm{Mg}$ and ${ }^{25} \mathrm{Mg}$ are predicted to be equal by TWW1995 and GP2001, but ALC2001 predict a higher value of ${ }^{26} \mathrm{Mg} /{ }^{25} \mathrm{Mg}$.

Our measured $\mathrm{Mg}$ isotopic ratios ${ }^{25} \mathrm{Mg} /{ }^{24} \mathrm{Mg}$ and ${ }^{26} \mathrm{Mg} /{ }^{24} \mathrm{Mg}$ are consistently higher than the predictions. The discrepancy is unlikely to stem from incorrect values for $[\mathrm{Fe} / \mathrm{H}]$, as errors exceeding 0.6 dex would be required, and in Paper I we showed that our derived metallicities agreed with published values. One possibility for accounting for the low predictions is that the yields from massive stars may underestimate the production of ${ }^{25} \mathrm{Mg}$ and ${ }^{26} \mathrm{Mg}$. In our current sample there is evidence that below $[\mathrm{Fe} / \mathrm{H}]=-1.5$ the $\mathrm{Mg}$ isotope ratios are higher than the predicted plateau. Observations of more $\mathrm{Mg}$ isotopic ratios at low metallicities, around the predicted plateau, would prove a powerful tool for testing theoretical yields from massive, metal-poor stars. Another possibility for explaining the low predictions for ${ }^{25} \mathrm{Mg} /{ }^{24} \mathrm{Mg}$ and ${ }^{26} \mathrm{Mg} /{ }^{24} \mathrm{Mg}$, one previously raised by Gay \& Lambert (2000), is that there is an additional source of the minor $\mathrm{Mg}$ isotopes. AGB stars can produce the neutron-rich isotopes, and their role is discussed in $\S 4.3$. We stress that none of the models include the contribution to ${ }^{25} \mathrm{Mg}$ and ${ }^{26} \mathrm{Mg}$ provided by the ejecta from intermediate-mass AGB stars. Therefore, the model predic- tions should be regarded as a lower limit to the observed $\mathrm{Mg}$ isotopic ratios. Indeed, these predictions provide a good fit to the lower envelope.

We reemphasize a point made by Gay \& Lambert (2000) regarding the predicted $\mathrm{Mg}$ isotopic ratios. The assumed mass cut for Type II supernovae affects the amount of $\mathrm{Fe}$ that is ejected, while the amounts of lighter elements ejected, including $\mathrm{Mg}$, are unaffected by the position of the mass cut. Adjustments to the mass cut have the effect of translating the predicted value of ${ }^{25} \mathrm{Mg} /{ }^{24} \mathrm{Mg}$ and ${ }^{26} \mathrm{Mg} /{ }^{24} \mathrm{Mg}$ along the $[\mathrm{Fe} / \mathrm{H}]$ axis. Both GP2000 and ALC2001 take the TWW1995 suggestion that the Fe yields from Type II supernovae need to be reduced by a factor of 2 in order to match observations. This corresponds to a factor of 0.3 in the log, and translating the TWW1995 predicted curve to lower metallicity effectively superimposes all predictions shown in Figures 8 and 9. Likewise, the assumed ratio of Type II to Type Ia supernovae exerts control over the shape of the predicted curve. Type II supernovae synthesize and eject the $\mathrm{Mg}$ isotopes and iron, whereas Type Ia supernovae return iron to the interstellar medium (ISM). Most nucleosynthetic predictions show that Type Ia supernovae produce 1 or 2 orders of magnitude less Mg than Type II supernovae. Different IMFs offer distinct ratios of Type II to Type Ia supernovae, affecting both the onset of the increase in the isotopic ratios and the slope of the predicted curve. We note that such adjustments cannot explain the scatter in the isotopic ratios and would adversely affect the fits to the observed run of elemental abundances (e.g., $\mathrm{Mg}, \mathrm{Si}$, and $\mathrm{Ca}$ ) with respect to $\mathrm{Fe}$.

\subsection{Role of $A G B$ Stars}

None of the predictions from the Galactic chemical evolution models take into account the yields of $\mathrm{Mg}$ from $\mathrm{AGB}$ stars. In all of the papers describing the models, a discussion is included to acknowledge and recognize that intermediatemass AGB stars can produce ${ }^{25} \mathrm{Mg}$ and ${ }^{26} \mathrm{Mg}$. Sufficiently massive AGB stars have He shells that can reach temperatures $\left(\sim 300 \times 10^{6} \mathrm{~K}\right)$ at which the neutron source, ${ }^{22} \mathrm{Ne}(\alpha, n)^{25} \mathrm{Mg}$, is activated. Hot bottom burning can take place if the base of the convective envelope reaches the top of the $\mathrm{H}$ shell. If hot bottom burning occurs at temperatures exceeding $\sim 90 \times 10^{6} \mathrm{~K}$, the $\mathrm{Mg}$-Al chain can deplete ${ }^{24} \mathrm{Mg}$ to produce ${ }^{25} \mathrm{Mg}$ and ${ }^{26} \mathrm{Mg}$. Karakas \& Lattanzio (2003) have shown that $6 M_{\odot} \mathrm{AGB}$ stars with $[\mathrm{Fe} / \mathrm{H}]=-0.7$ produce an envelope with large amounts of ${ }^{25} \mathrm{Mg}$ and ${ }^{26} \mathrm{Mg}$ and essentially no ${ }^{24} \mathrm{Mg}$. This envelope is then ejected as the star becomes a planetary nebula, enriching the ISM with the neutron-rich $\mathrm{Mg}$ isotopes. In contrast to supernovae, which eject material at high velocities $\left(\sim 5000 \mathrm{~km} \mathrm{~s}^{-1}\right)$, AGB stars eject gas at low velocities $\left(\sim 10 \mathrm{~km} \mathrm{~s}^{-1}\right)$. The ejecta from AGB stars may therefore be confined to a more localized region, and inhomogeneities in the ISM are possible until the ISM is mixed by supernovae. The timescale for star formation relative to mixing in the ISM is then an important factor. Stars formed from a region of the ISM recently polluted by massive AGB stars will therefore be enriched in ${ }^{25} \mathrm{Mg},{ }^{26} \mathrm{Mg}$, and $s$-process elements.

Gay \& Lambert (2000) measured overabundances of ${ }^{25} \mathrm{Mg}$ and ${ }^{26} \mathrm{Mg}$ in a number of stars known to have higherthan-usual abundances of $s$-process elements. These elemental and isotopic compositions are consistent with the idea that the stars' natal clouds were enriched by ejecta from intermediate-mass AGB stars. One star in our sample, 
$\mathrm{BD}+05^{\circ} 3640$, has already been shown to be a $\mathrm{CH}$ star (Tomkin \& Lambert 1999). We find that it has high ratios of ${ }^{25} \mathrm{Mg} /{ }^{24} \mathrm{Mg}$ and ${ }^{26} \mathrm{Mg} /{ }^{24} \mathrm{Mg}$ compared to other stars of similar metallicity. Mass transfer from a companion AGB star, now an unseen white dwarf, changed BD $+05^{\circ} 3640$ into a $\mathrm{CH}$ star and produced the observed enhancements in ${ }^{25} \mathrm{Mg},{ }^{26} \mathrm{Mg}$, and $s$-process elements.

Our Mg isotopic ratios exceed the predictions calculated under the assumption that only massive stars produce ${ }^{25} \mathrm{Mg}$ and ${ }^{26} \mathrm{Mg}$. Inclusion of the yields of ${ }^{25} \mathrm{Mg}$ and ${ }^{26} \mathrm{Mg}$ from AGB stars would increase the predicted ratio of ${ }^{25} \mathrm{Mg} /{ }^{24} \mathrm{Mg}$ and ${ }^{26} \mathrm{Mg} /{ }^{24} \mathrm{Mg}$ and introduce a scatter that would qualitatively match our measured ratios. Busso et al. (2001) have shown that $5 M_{\odot}$ AGB stars produce considerable amounts of $\mathrm{Y}$ and $\mathrm{Zr}$ (and other $s$-process elements), in addition to the neutron-rich $\mathrm{Mg}$ isotopes. Busso et al. did not include hot bottom burning in their models. Comparison of the Busso et al. and Karakas \& Lattanzio (2003) yields shows that hot bottom burning is the dominant production site of the minor $\mathrm{Mg}$ isotopes in $5 M_{\odot}$ AGB stars. Quantitative yields from AGB stars for a variety of masses and metallicities need to be incorporated into comprehensive models of Galactic chemical evolution to provide thorough predictions of the evolution of the $\mathrm{Mg}$ isotopes. J. Simmerer et al. (2003, private communication) are also investigating the role of AGB stars from a different perspective. From measurements of the ratio of $\mathrm{La}$ ( $s$-process) to $\mathrm{Eu}$ ( $r$-process), their data suggest that the contribution of AGB stars to Galactic chemical evolution commences around $[\mathrm{Fe} / \mathrm{H}] \simeq$ -2.0 . This evidence supports the results of previous studies based on the abundances of $\mathrm{Ba}$ and $\mathrm{Eu}$ (e.g., Spite \& Spite 1978; Burris et al. 2000).

We performed a back-of-the-envelope calculation to investigate whether enhancements of $\mathrm{Zr}$ (a representative light $s$-process element) should be detectable in stars with high ratios of ${ }^{26} \mathrm{Mg} /{ }^{24} \mathrm{Mg}$. In this simple exercise, we assumed that the enrichment of ${ }^{26} \mathrm{Mg}$ is a consequence of pollution by AGB ejecta. Taking the Karakas \& Lattanzio $\mathrm{Mg}$ yields for a $5 M_{\odot}, Z=0.004 \mathrm{AGB}$ star, we found that a mix of 200 parts ambient material to 1 part AGB ejecta was required to produce ${ }^{26} \mathrm{Mg} /{ }^{24} \mathrm{Mg}=0.3$. Using the Busso et al. (2001) $\mathrm{Zr}$ yields for a $5 M_{\odot}, Z=0.002 \mathrm{AGB}$ star, we estimate that a mix of 200 parts ambient material to 1 part AGB ejecta increases the value of $[\mathrm{Zr} / \mathrm{Fe}]$ by only $0.06 \mathrm{dex}$. Therefore, at high metallicity, ejecta from AGB stars that have experienced hot bottom burning may increase the ratio of ${ }^{26} \mathrm{Mg} /{ }^{24} \mathrm{Mg}$ to the highest levels observed without producing a detectable enhancement of $s$-process elements.

Intermediate-mass AGB stars are expected to eject $\mathrm{N}$-rich material along with the neutron-rich $\mathrm{Mg}$ isotopes and $s$-process elements. HD 25329 is overabundant in $\mathrm{N}$ (Carbon et al. 1987), s-process elements (Beveridge \& Sneden 1994), and ${ }^{25} \mathrm{Mg}$ and ${ }^{26} \mathrm{Mg}$ (Gay \& Lambert 2000). Ventura, D’Antona, \& Mazzitelli (2002) calculated yields from low-metallicity AGB stars, including the effects of hot bottom burning. They find enhancements of $\mathrm{N}$ by a factor of 30 despite "conservative assumptions on the third dredge-up." However, repeating the above exercise of mixing 200 parts ambient material to 1 part AGB ejecta would not produce a significant enhancement of N. Since stars are known to be N-rich and overabundant in ${ }^{25} \mathrm{Mg}$ and ${ }^{26} \mathrm{Mg}$, the models may require more realistic assumptions about the third dredge-up or a smaller dilution factor.
We have unveiled the crucial role played by AGB stars in the chemical evolution of globular cluster NGC 6752 (Yong et al. 2003). This cluster contains stars that display a significant star-to-star abundance variation in $\mathrm{O}, \mathrm{Na}, \mathrm{Mg}$, and $\mathrm{Al}$. At one extreme of the abundance variation, "normal " stars have elemental compositions similar to field stars at the same metallicity $([\mathrm{Fe} / \mathrm{H}]=-1.6)$ that are well explained by ejecta from metal-poor, massive stars dying as Type II supernovae. However, the $\mathrm{Mg}$ isotopic ratios found in these normal cluster giants $\left({ }^{24} \mathrm{Mg}:{ }^{25} \mathrm{Mg}:{ }^{26} \mathrm{Mg} \simeq 80: 10: 10\right)$ exceed predictions $\left({ }^{24} \mathrm{Mg}:{ }^{25} \mathrm{Mg}:{ }^{26} \mathrm{Mg} \simeq 98: 1: 1\right)$ from massive stars. We note that at $[\mathrm{Fe} / \mathrm{H}]=-1.6$, ratios of $80: 10: 10$ match the upper envelope in Figures 8 and 9. Zero-metallicity AGB stars can raise the low abundances of ${ }^{25} \mathrm{Mg}$ and ${ }^{26} \mathrm{Mg}$ provided by the supernovae to the high levels observed in the normal stars. At the other extreme of the abundance variation, "polluted" stars are underabundant in $\mathrm{O}$ and $\mathrm{Mg}$ and overabundant in $\mathrm{Na}$ and $\mathrm{Al}$, with respect to the normal stars, and have ${ }^{24} \mathrm{Mg}:{ }^{25} \mathrm{Mg}:{ }^{26} \mathrm{Mg} \simeq$ $60: 10: 30$. For $[\mathrm{Fe} / \mathrm{H}]=-1.6$, ratios of $60: 10: 30$ greatly exceed the highest values in Figures 8 and 9. We refer to these stars as polluted because their compositions are well explained by ejecta from AGB stars of the same metallicity as the cluster.

\subsection{Which Stellar Population?}

In $\S 2$, we mentioned that our sample was kinematically selected so that a considerable fraction have Galactic space velocities and $\mathrm{Fe}$ abundances indicative of halo or thickdisk stars. At low metallicities, the models predict the $\mathrm{Mg}$ isotopes for halo stars. At high metallicities, the predicted $\mathrm{Mg}$ isotopic ratios are for the thin disk, whereas our kinematically selected sample of higher metallicity stars likely contain a mix of thick- and thin-disk stars. In effect, we may be comparing two different stellar populations: predictions for thin-disk stars and measurements from thick-disk stars. In Figures 8 and 9, the solar $\mathrm{Mg}$ isotopic ratio appears rather low relative to the isotopic ratios in stars with similar metallicities. Is this due to the sample's bias to thick-disk stars? The seven stars observed in 2003 February were selected to be dwarfs of around solar metallicity with thindisk kinematics. All seven stars had Mg isotope ratios similar to the solar values (see Table 1). This suggests that the Sun is a representative member of the thin disk.

Gilmore \& Reid (1983) first measured the scale height of the Galactic thick disk, and since then many efforts have been devoted to characterizing the thick-disk population. Recent work (e.g., Fuhrmann 1998; Prochaska et al. 2000; Feltzing, Bensby, \& Lundström 2003; Mashonkina et al. 2003; Reddy et al. 2003) indicates that below $[\mathrm{Fe} / \mathrm{H}]=$ -0.3 , thick-disk stars are overabundant in $\mathrm{Mg}$ $([\mathrm{Mg} / \mathrm{Fe}]=0.4)$ relative to thin-disk stars $([\mathrm{Mg} / \mathrm{Fe}]=0.1)$. This difference (also evident for $\mathrm{Ti}, \mathrm{Ca}$, and other elements) has been attributed to the importance of Type II supernovae relative to Type Ia supernovae in the chemical history of the thick-disk population. The high ratios of $[\alpha / \mathrm{Fe}]$ at high metallicities are a defining characteristic of the thick-disk population. Not only are these thick-disk stars chemically different from the thin-disk stars, consideration of their space velocities also reveals systematic differences.

Figure 13 shows the effect of our selection criteria, which are biased against thin-disk stars. The Toomre diagram of our stars shows that only 15 of the 75 stars are likely 


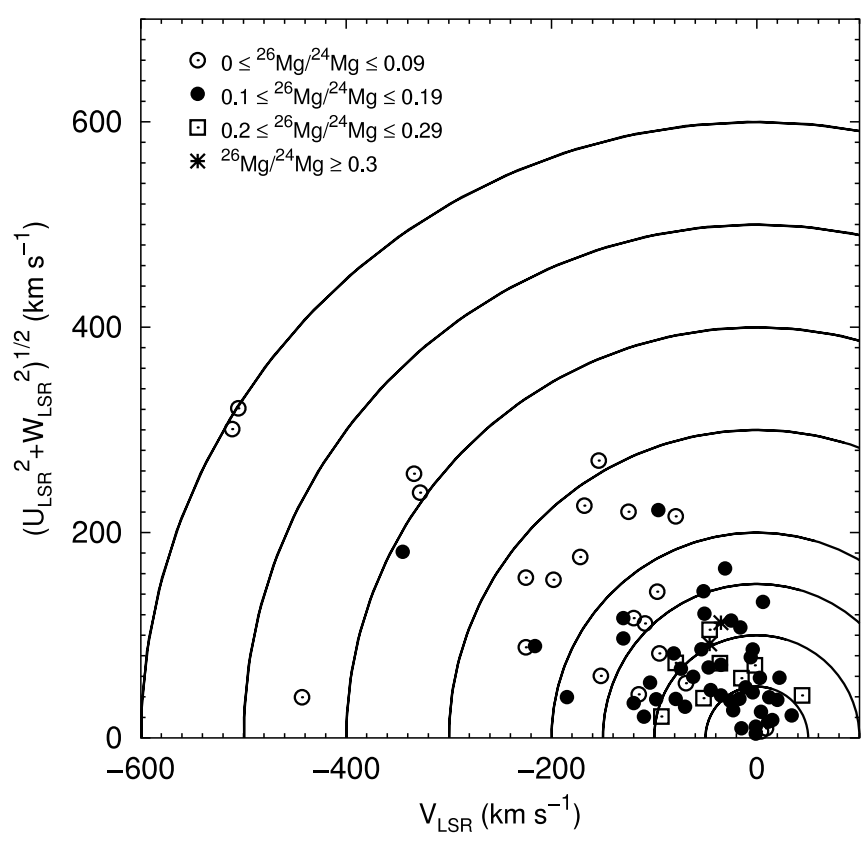

FIG. 13.- Toomre diagram for our stars and the Gay \& Lambert (2000) sample. The concentric circles represent lines of constant kinetic energy. The open circles represent stars with $0 \leq{ }^{26} \mathrm{Mg} /{ }^{24} \mathrm{Mg} \leq 0.09$, the closed circles represent stars with $0.1 \leq{ }^{26} \mathrm{Mg} /{ }^{24} \mathrm{Mg} \leq 0.19$, the open squares represent stars with $0.2 \leq{ }^{26} \mathrm{Mg} /{ }^{24} \mathrm{Mg} \leq 0.29$, and the asterisks represent stars with ${ }^{26} \mathrm{Mg} /{ }^{24} \mathrm{Mg} \geq 0.3$. Note the absence of stars with ${ }^{26} \mathrm{Mg} /{ }^{24} \mathrm{Mg} \geq 0.2$ and space velocities within $U_{\mathrm{LSR}}^{2}+V_{\mathrm{LSR}}^{2}+W_{\mathrm{LSR}}^{2} \leq$ $\left(50 \mathrm{~km} \mathrm{~s}^{-1}\right)^{2}$. The majority of stars lie beyond $U_{\mathrm{LSR}}^{2}+V_{\mathrm{LSR}}^{2}+W_{\mathrm{LSR}}^{2} \geq$ $\left(50 \mathrm{~km} \mathrm{~s}^{-1}\right)^{2}$, demonstrating that we have deliberately selected against stars with thin-disk kinematics.

thin-disk stars, with $U^{2}+V^{2}+W^{2} \leq\left(50 \mathrm{~km} \mathrm{~s}^{-1}\right)^{2}$. All stars with ${ }^{26} \mathrm{Mg} /{ }^{24} \mathrm{Mg} \geq 0.2$ lie in the ranges

$$
\begin{aligned}
& -150 \mathrm{~km} \mathrm{~s}^{-1} \leq V \leq-50 \mathrm{~km} \mathrm{~s}^{-1}, \\
& 0 \mathrm{~km} \mathrm{~s}^{-1} \leq\left(U^{2}+W^{2}\right)^{1 / 2} \leq 150 \mathrm{~km} \mathrm{~s}^{-1},
\end{aligned}
$$

which is the region of the Toomre diagram from which candidate thick-disk stars are selected (e.g., Fuhrmann $2000^{3}$; Feltzing et al. 2003).

In Figure 14, we separate our stars according to their kinematics and plot the ratio ${ }^{26} \mathrm{Mg} /{ }^{24} \mathrm{Mg}$ versus $[\mathrm{Fe} / \mathrm{H}]$. A striking trend is evident when we consider only those stars with $\left(U^{2}+V^{2}+W^{2}\right)^{1 / 2} \leq 50 \mathrm{~km} \mathrm{~s}^{-1}$. For these stars, the $\mathrm{Mg}$ isotope ratio ${ }^{26} \mathrm{Mg} /{ }^{24} \mathrm{Mg}$ is in excellent agreement with the predictions. The small scatter about the predicted curve may be entirely attributable to the measurement uncertainties. It should be noted that the scatter in abundances (i.e., $[\mathrm{X} / \mathrm{Fe}])$ at a given $[\mathrm{Fe} / \mathrm{H}]$ is also very small for thin-disk stars for $\mathrm{X}=\mathrm{C}$ to $\mathrm{Eu}$ (Reddy et al. 2003). These stars are almost certainly members of the thin disk based on their kinematics and metallicities. Next, we consider stars with $50 \mathrm{~km} \mathrm{~s}^{-1}<$ $\left(U^{2}+V^{2}+W^{2}\right)^{1 / 2} \leq 100 \mathrm{~km} \mathrm{~s}^{-1}$. While some of the sample are in good agreement with the predictions, others exceed the predictions. The stars in this sample extend to lower metallicities than the $\left(U^{2}+V^{2}+W^{2}\right)^{1 / 2} \leq 50 \mathrm{~km} \mathrm{~s}^{-1}$ sample. Based on the kinematics and metallicities, these stars are a mix of thin- and thick-disk stars. Now we consider stars with

\footnotetext{
${ }^{3}$ Available at http://www.xray.mpe.mpg.de/ fuhrmann.
}

$100 \mathrm{~km} \mathrm{~s}^{-1}<\left(U^{2}+V^{2}+W^{2}\right)^{1 / 2} \leq 150 \mathrm{~km} \mathrm{~s}^{-1}$. A small fraction of the sample show good agreement with the predictions, with the remaining stars exceeding the predictions. The stars in this kinematic range exhibit the largest spread in the $\mathrm{Mg}$ isotope ratios and the largest spread in $[\mathrm{Fe} / \mathrm{H}]$. At present, the scatter in elemental abundances (i.e., $[\mathrm{X} / \mathrm{Fe}]$ ) at a given $[\mathrm{Fe} / \mathrm{H}]$ is not well known for the thick disk. It is small for halo stars. These stars are a mix of halo and thick-disk stars, with the possibility that some stars may belong to the high-velocity tail of the thin-disk distribution. Finally, we consider the stars with $\left(U^{2}+V^{2}+W^{2}\right)^{1 / 2}>150 \mathrm{~km} \mathrm{~s}^{-1}$. About half the sample are in good agreement with the predictions, while the other half exceed the predictions. These stars span a smaller range in $[\mathrm{Fe} / \mathrm{H}]$ than the $100 \mathrm{~km} \mathrm{~s}^{-1}<$ $\left(U^{2}+V^{2}+W^{2}\right)^{1 / 2} \leq 150 \mathrm{~km} \mathrm{~s}^{-1}$ sample, and the total spread in $\mathrm{Mg}$ isotope ratios is also smaller (the highest value belongs to the $\mathrm{CH}$ star $\mathrm{BD}+05^{\circ} 3640$ ). This sample contains halo stars and possibly some thick-disk stars.

Stars we unambiguously identify as thin-disk members have $\mathrm{Mg}$ isotope ratios in excellent agreement with predictions, with little scatter about the predicted curve. When we consider a sample of stars that contains a mix of thin-disk, thick-disk, and halo stars, the $\mathrm{Mg}$ isotope ratios show a large scatter, including stars that greatly exceed the predictions. The high $\mathrm{Mg}$ isotopic ratios may come from thickdisk stars. Let us assume that Type II supernovae are almost entirely responsible for the iron abundance, with little, if any, contribution from Type Ia supernovae. Under this assumption, Type II supernovae are responsible for the $\mathrm{Fe}$ and Mg. From the Woosley \& Weaver (1995) yields, metalrich Type II supernovae produce significant amounts of ${ }^{25} \mathrm{Mg}$ and ${ }^{26} \mathrm{Mg}$ relative to ${ }^{24} \mathrm{Mg}$, so it may be reasonable to expect high ratios of ${ }^{25} \mathrm{Mg} /{ }^{24} \mathrm{Mg}$ and ${ }^{26} \mathrm{Mg} /{ }^{24} \mathrm{Mg}$ in thickdisk stars. It would be useful to obtain predictions of the $\mathrm{Mg}$ isotope ratios from a Galactic chemical evolution model that neglects the contribution from Type Ia supernovae. In such a model, do stars with $[\mathrm{Fe} / \mathrm{H}] \simeq 0$ have $\mathrm{Mg}$ isotopic ratios similar to the values we measure?

\subsection{Scatter}

Attributing the scatter in $\mathrm{Mg}$ isotopic ratios at a fixed $[\mathrm{Fe} / \mathrm{H}]$ to different ratios of Type II supernovae to AGB ejecta in the star-forming interstellar clouds is an appealing idea. Yet there is an alternative that may be as plausible and appealing.

The yield of ${ }^{25} \mathrm{Mg}$ and ${ }^{26} \mathrm{Mg}$ (relative to ${ }^{24} \mathrm{Mg}$ ) in Type II supernova ejecta is firmly expected to be dependent on the stars' initial composition, as explained above. (We assume here that the composition and initial mass of a star are the key parameters. If other factors - angular momentum, for example - are relevant, the argument that follows is weakened.) The yield of ${ }^{24} \mathrm{Mg}$ is only slightly dependent on the initial composition of the massive stars. Relative to the ${ }^{24} \mathrm{Mg}$ yield, yields for some elements will vary little with initial metallicity, e.g., $\mathrm{Si}$ and $\mathrm{Ca}$, i.e., $\alpha$-elements. Yields for other elements will be metallicity-dependent, e.g., $\mathrm{Na}$ and $\mathrm{Al}$.

Consider now the case in which stars form in interstellar clouds contaminated to differing degrees with Type II supernova ejecta. If the ejecta provided to all clouds come from stars of the same initial composition, the $\mathrm{Mg} / \mathrm{H}$ ratio of the stars will be high for stars from severely contaminated clouds and lower for those stars from lightly contaminated 


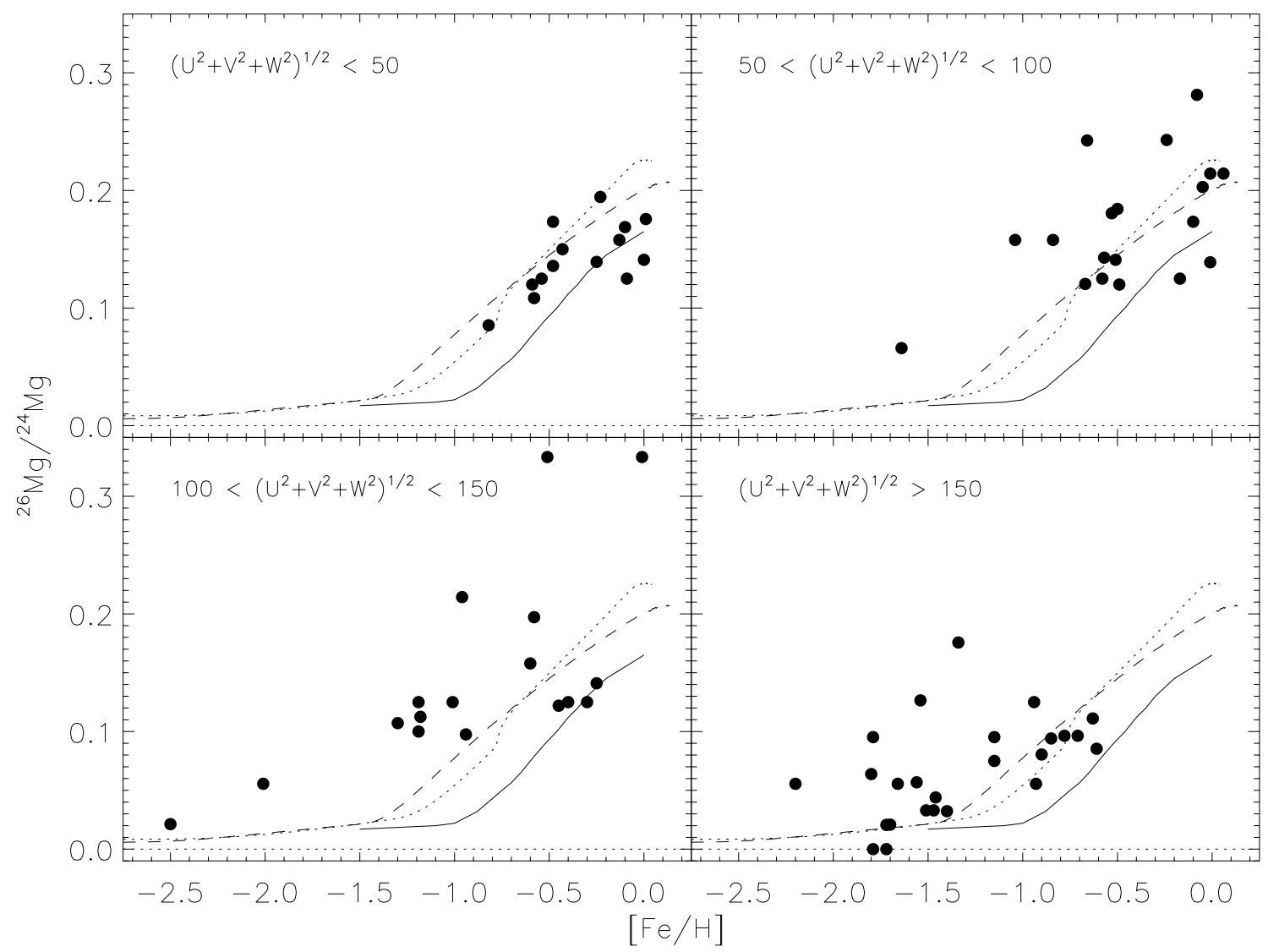

FIG. 14. - Isotopic ratio ${ }^{26} \mathrm{Mg} /{ }^{24} \mathrm{Mg}$ vs. $[\mathrm{Fe} / \mathrm{H}]$, grouped by space velocities. We plot $\left(U^{2}+V^{2}+W^{2}\right)^{1 / 2} \leq 50($ top left $), 50<\left(U^{2}+V^{2}+W^{2}\right)^{1 / 2} \leq 100$ (top right), $100<\left(U^{2}+V^{2}+W^{2}\right)^{1 / 2} \leq 150$ (bottom left), and $\left(U^{2}+V^{2}+W^{2}\right)^{1 / 2}>150$ (bottom right), where all velocities are in $\mathrm{km} \mathrm{s} \mathrm{s}^{-1}$. In each panel we plot the TWW1995 (solid line), GP2000 (dashed line), and ALC2001 (dotted line) predictions.

clouds. Abundance ratios, such as $\mathrm{Mg} / \mathrm{Si}$ and ${ }^{26} \mathrm{Mg} /{ }^{24} \mathrm{Mg}$, may be almost independent of the degree of contamination.

More likely than this simplest of scenarios is the case of clouds contaminated to differing degrees by supernova ejecta from stars of different initial metallicity. The stars born in these clouds will show different $\mathrm{Mg} / \mathrm{H}$ ratios, but abundance ratios, such as $\mathrm{Mg} / \mathrm{Si}$, may vary little across the stellar sample, because their respective yields are only weakly dependent on initial metallicity of the supernovae's progenitors. In contrast, a ratio like ${ }^{26} \mathrm{Mg} /{ }^{24} \mathrm{Mg}$, comprised of species whose relative yields are metallicity-dependent, will vary across a sample of stars of the same $\mathrm{Mg} / \mathrm{H}$. This scenario is presumably most relevant to clouds of low initial metallicity and least relevant to clouds of high metallicity.

An assessment of the applicability of this simple idea as a partial explanation for the scatter in Figures 8 and 9 must await the full abundance analyses of our stars. A few comments are offered.

For thin-disk stars, the scatter in the $\mathrm{Mg}$ isotopic ratios is not detectably greater than the errors of measurement. This is not a surprising result, because the stars are among the most metal-rich of our sample and, hence, are formed from clouds contaminated by several generations of supernovae. More directly, elemental abundance ratios at a given $[\mathrm{Fe} / \mathrm{H}]$ show no intrinsic scatter across a large sample of thin-disk main-sequence stars (Reddy et al. 2003). Elements investigated include those whose yields from Type II supernovae are dependent on the massive stars' initial composition.
Stars with $[\mathrm{Fe} / \mathrm{H}] \leq-1.5$ show a spread in the $\mathrm{Mg}$ isotopic ratios from the very low values predicted from metal-poor, massive stars to values (say, ${ }^{26} \mathrm{Mg} /{ }^{24} \mathrm{Mg} \sim$ 0.05) representative of supernovae ejecta from stars with metallicityhigher than that observed in our stars. Our simple scenario implies that the supernovae feeding these stars' natal clouds had compositions with $Z \simeq 0.01$. It may be possible to test this conjecture using abundance ratios such as $\mathrm{Na} / \mathrm{Mg}$, as indicated above. By using several such ratios, it should be possible also to differentiate between $\mathrm{Mg}$ isotopic ratios resulting from our scenario and those attributable to contamination of natal clouds by ejecta from AGB stars.

The scenario cannot, however, account for the stars with the highest $\mathrm{Mg}$ isotopic ratios, say ${ }^{26} \mathrm{Mg} /{ }^{24} \mathrm{Mg} \geq 0.2$. These demand either unexpectedly high isotopic ratios from Type II supernovae or contamination of the star (or its natal cloud) by ejecta from an intermediate-mass AGB star. Again, close scrutiny of a suite of elemental abundances should be helpful.

\subsection{Nonzero Ratios at Low Metallicity}

We claimed that six stars below $[\mathrm{Fe} / \mathrm{H}]=-1.5$ have nonzero $\mathrm{Mg}$ isotope ratios, ${ }^{26} \mathrm{Mg} /{ }^{24} \mathrm{Mg}>0.05$. Our uncertainties in the isotope ratios include errors in the model parameters and errors in the fits, and even allowing for these uncertainties, our $\mathrm{Mg}$ isotope ratios still exceed the 
predictions (Figs. 8 and 9). At low metallicities, the predictions from all three models are essentially identical, since they all make use of the Woosley \& Weaver (1995) massivestar yields. As a consequence of primary production of the neutron-rich $\mathrm{Mg}$ isotopes, the models predict a low, but nonzero, plateau in the ratios ${ }^{25} \mathrm{Mg} /{ }^{24} \mathrm{Mg}$ and ${ }^{26} \mathrm{Mg} /{ }^{24} \mathrm{Mg}$. While more observations are required to confirm that the measurements exceed the predicted plateau, two possibilities exist. Either the supernovae yields are in error or intermediate-mass stars contribute ejecta to low-metallicity gas.

If intermediate-mass stars contribute their ejecta to lowmetallicity gas, then the ratio ${ }^{26} \mathrm{Mg} /{ }^{24} \mathrm{Mg}$ will also exceed the predictions discussed above. These AGB stars may also leave another signature, enhancements in $s$-process elements, that may be used to identify the origin of the enhanced ${ }^{25} \mathrm{Mg}$ and ${ }^{26} \mathrm{Mg}$. Ultimately, in order to fully explore either scenario, more measurements of $\mathrm{Mg}$ isotope ratios at low metallicities are essential.

\section{CONCLUDING REMARKS}

Our $61 \mathrm{Mg}$ isotopic abundance ratios confirm the decrease in ${ }^{25} \mathrm{Mg} /{ }^{24} \mathrm{Mg}$ and ${ }^{26} \mathrm{Mg} /{ }^{24} \mathrm{Mg}$ with decreasing $[\mathrm{Fe} / \mathrm{H}]$. We compared the evolution of the $\mathrm{Mg}$ isotopes with predictions from models of Galactic chemical evolution in which the $\mathrm{Mg}$ isotopes are the product of massive stars. The comparison highlights that, in general, our observed ratios exceed the predictions. Inclusion of the ejecta from AGB stars, known to synthesize and eject the neutron-rich minor $\mathrm{Mg}$ isotopes, may reconcile the observations and predictions. There is a scatter in the observed ratios that exceeds the measurement uncertainties, and the scatter increases with increasing metallicity. Our kinematically selected sample may contain a considerable fraction of thick-disk stars. The comparison between our observed ratios and the predicted ratios for the disk is, then, effectively a comparison between two different stellar populations. Regardless of whether we have observed thin- and/or thick-disk stars, around solar metallicity the $\mathrm{Mg}$ isotope ratios range from solar to more than twice the solar value. $\mathrm{Mg}$ isotope ratios in thin-disk stars (identified kinematically) show an excellent agreement with the predictions, with little scatter about the predicted curve.

At high metallicities, are AGB stars responsible for the large abundances of ${ }^{25} \mathrm{Mg}$ and ${ }^{26} \mathrm{Mg}$ ? What fraction of the stars with large abundances of ${ }^{25} \mathrm{Mg}$ and ${ }^{26} \mathrm{Mg}$ are thick-disk stars devoid of products from Type Ia supernovae? Do these stars show peculiarities in any other elemental abundances? We have commenced an analysis of the abundances of various $\alpha$-, iron peak, and neutron-capture elements, beginning with the stars that have ${ }^{26} \mathrm{Mg} /{ }^{24} \mathrm{Mg}>0.2$. Only $\mathrm{BD}+05^{\circ} 3640$ shows enhancements in $s$-process elements, and Tomkin \& Lambert (1999) have already demonstrated that this is a CH star. Presumably, thick-disk stars would be readily identified by an excess in $\alpha$-elements, whereas stars contaminated by AGB ejecta may be marked by overabundances in $s$-process elements. At this preliminary stage, we do not see either signature in the stars. Although isotopic ratios are unaffected, uncertainties in our stellar parameters may mask subtle differences in elemental abundances.

Below some critical metallicity, the AGB stars will not have had time to evolve and eject their material into the ISM. Metal-poor dwarfs formed from early material will then have compositions reflecting nucleosynthesis in prior generations of massive, metal-poor stars. Observations of isotopic ratios in the range $[\mathrm{Fe} / \mathrm{H}]<-2.0$ offer the opportunity to test predicted yields from massive, metal-poor stars. Is there a plateau in the $\mathrm{Mg}$ isotopic ratios at low metallicities, as predicted from the models? If the plateau exists, does it agree with the predicted value? There is evidence that our observed ratios are higher than predictions below $[\mathrm{Fe} / \mathrm{H}]=-1.5$. Further observations of $\mathrm{Mg}$ isotopes in the range $[\mathrm{Fe} / \mathrm{H}]<-2.0$ will also show when intermediatemass AGB stars begin contributing to the Galactic chemical evolution. These observations offer the chance to refine our understanding of stellar nucleosynthesis. However, these cool, metal-poor stars must first be identified. We are continuing to search for low-luminosity, cool, metal-poor dwarfs.

Not only should the search for metal-poor dwarfs be continued, but a parallel investigation should be made of the appropriateness of the standard method of analysisclassical model atmospheres, LTE, etc. One simple test would be to measure by the standard methods the Mg isotope ratio in main-sequence stars from an open cluster, say the Hyades. In such a sample, it is plausible to assume that the stars are chemically homogeneous. A variation of the $\mathrm{Mg}$ isotope ratios with effective temperature would then signal the presence of systematic errors afflicting the standard methods of analysis. Then the challenge would be to identify those errors.

We thank Amanda Karakas and Maurizio Busso for providing comprehensive yields from intermediate-mass AGB stars. We thank Javier Labay and Nicolas Prantzos for providing their predictions in electronic form. D. Y. thanks Amanda Karakas, Bacham Reddy, Carlos Allende Prieto, Gajendra Pandey, Jennifer Simmerer, John Lattanzio, and Yeshe Fenner for many helpful discussions. D. L. L. and D. Y. acknowledge support from the Robert A. Welch Foundation of Houston, Texas. I. I. I.'s funding support is from NASA, through Hubble Fellowship grant HST-HF01151.01-A from the Space Telescope Science Institute, operated by AURA, Inc., under NASA contract NAS526555. This research has made use of the SIMBAD database, operated at CDS, Strasbourg, France, and NASA's Astrophysics Data System.
Alibés, A., Labay, J., \& Canal, R. 2001, A\&A, 370, 1103 (ALC2001)

Arnett, W. D., \& Thielemann, F.-K. 1985, ApJ, 295, 589

Barbuy, B. 1985, A\&A, 151, 189

Barbuy, B., Spite, F., \& Spite, M. 1987, A\&A, 178, 199

Bernath, P. F., Black, J. H., \& Brault, J. W. 1985, ApJ, 298, 375

Beveridge, C. R., \& Sneden, C. 1994, AJ, 108, 285

Boesgaard, A. M. 1968, ApJ, 154, 185

Burris, D. L., Pilachowski, C. A., Armandroff, T. E., Sneden, C., Cowan, J. J., \& Roe, H. 2000, ApJ, 544, 302

Busso, M., Gallino, R., Lambert, D. L., Travaglio, C., \& Smith, V. V. 2001, ApJ, 557, 802

\section{REFERENCES}

Carbon, D. F., Barbuy, B., Kraft, R. P., Friel, E. D., \& Suntzeff, N. B. 1987, PASP, 99, 335

Carney, B. W., Latham, D. W., Laird, J. B., \& Aguilar, L. A. 1994, AJ, 107, 2240

Cottrell, P. L. 1978, ApJ, 223, 544

Dehnen, W., \& Binney, J. J. 1998, MNRAS, 298, 387

Edvardsson, B., Andersen, J., Gustafsson, B., Lambert, D. L., Nissen, P. E., \& Tomkin, J. 1993, A\&A, 275, 101

Feltzing, S., Bensby, T., \& Lundström, I. 2003, A\&A, 397, L1

Forestini, M., \& Charbonnel, C. 1997, A\&AS, 123, 241

Fuhrmann, K. 1998, A\&A, 338, 161 
Fuhrmann, K. 2000, A\&A, submitted

Fulbright, J. P. 2000, AJ, 120, 1841

Gay, P. L., \& Lambert, D. L. 2000, ApJ, 533, 260

Gilmore, G., \& Reid, N. 1983, MNRAS, 202, 1025

Goswami, A., \& Prantzos, N. 2000, A\&A, 359, 191 (GP2000)

Hauschildt, P. H., Allard, F., \& Baron, E. 1999, ApJ, 512, 377

Iben, I., Jr. 1975, ApJ, 196, 525

Karakas, A. I., \& Lattanzio, J. C. 2003, Publ. Astron. Soc. Australia, 20, 279

Kroupa, P., Tout, C. A., \& Gilmore, G. 1993, MNRAS, 262, 545

Lambert, D. L., Heath, J. E., Lemke, M., \& Drake, J. 1996, ApJS, 103, 183

Lambert, D. L., Mallia, E. A., \& Petford, A. D. 1971, MNRAS, 154, 265

Lambert, D. L., \& McWilliam, A. 1986, ApJ, 304, 436

Mashonkina, L., Gehren, T., Travaglio, C., \& Borkova, T. 2003, A\&A, 397,275

McWilliam, A., \& Lambert, D. L. 1988, MNRAS, 230, 573

McWilliam, A., Preston, G. W., Sneden, C., \& Searle, L. 1995, AJ, 109, 2757

Pilachowski, C. A., Sneden, C., \& Kraft, R. P. 1996, AJ, 111, 1689

Prochaska, J. X., Naumov, S. O., Carney, B. W., McWilliam, A., \& Wolfe, A. M. 2000, AJ, 120, 2513
Reddy, B. E., Tomkin, J., Lambert, D. L., \& Allende Prieto, C. 2003, MNRAS, 340, 304

Ryan, S. G., Norris, J. E., \& Beers, T. C. 1996, ApJ, 471, 254

Shetrone, M. D. 1996, AJ, 112, 2639

Sneden, C. 1973, ApJ, 184, 839

Spite, M., \& Spite, F. 1978, A\&A, 67, 23

Thielemann, F.-K., \& Arnett, W. D. 1985, ApJ, 295, 604

Timmes, F. X., Woosley, S. E., \& Weaver, T. A. 1995, ApJS, 98, 617 (TWW1995)

Tomkin, J., \& Lambert, D. L. 1980, ApJ, 235, 925

Tull, R. G., MacQueen, P. J., Sneden, C., \& Lambert, D. L. 1995, PASP, 107,251

Ventura, P., D’Antona, F., \& Mazzitelli, I. 2002, A\&A, 393, 215

Woosley, S. E., \& Weaver, T. A. 1995, ApJS, 101, 181

Yong, D., Grundahl, F., Lambert, D. L., Nissen, P. E., \& Shetrone, M. D. 2003, A\&A, 402, 985

Yong, D., \& Lambert, D. L. 2003a, PASP, 115, 22 (Paper I) 2003b, PASP, 115, 796 